\title{
Proof Systems for Message-Passing Process Algebras
}

\author{
M. Hennessy and H. Lin \\ School of Cognitive and Computing Sciences, University of Sussex, Brighton, UK
}

Keywords: Process algebra; Message passing; Bisimulation equivalence; Proof system; Completeness; Symbolic semantics

Abstract. We give sound and complete proof systems for a variety of bisimulation based equivalences over a message-passing process algebra. The process algebra is a generalisation of pure CCS where the actions consist of receiving and sending messages or data on communication channels; the standard prefixing operator a.p is replaced by the two operators $c$ ? x.p and c!e.p and in addition messages can be tested by a conditional construct. The various proof systems are parameterised on auxiliary proof systems for deciding on equalities or more general boolean identities over the expression language for data. The completeness of these proof systems are thus relative to the completeness of the auxiliary proof systems.

\section{Introduction}

In standard or pure process algebras processes are described in terms of their ability to perform atomic unanalysed actions. For example

$$
P \Longleftarrow a . P+\text { b.c. } P
$$

describes a process which can continually either perform the action $a$ or the sequence of actions $b, c$. By a message-passing process algebra we mean a process algebra in which these actions are given some structure; namely the reception or emission of data values on communication channels. Thus

$$
Q \Longleftarrow c \text { ? } x \text {. if } x \geq 0 \text { then } d ! x . Q \text { else } c !(x+1) \cdot Q
$$

Correspondence and offprint requests to: $\mathrm{M}$. Hennessy and $\mathrm{H}$. Lin, School of Cognitive and Computing Sciences, University of Sussex, Brighton BN1 9QH, UK. 
describes a process which can cyclically input a value along the channel $c$ and either output it along the channel $d$ unchanged or output its successor along $c$, depending on whether or not the value concerned is greater than or equal to 0 .

The standard approach to providing a semantic basis for these messagepassing algebras, advocated for example in [Mil89], is to translate them into an underlying pure algebra. The central feature of this translation, mapping $p$ to $\llbracket p \rrbracket$, is that the input expression $c$ ?x.p is mapped into the term

$$
\sum_{v \in V a l} c ? v \cdot \llbracket p[v / x] \rrbracket
$$

where $\mathrm{Val}$ is the domain of all data values. Thus the translation of the process $Q$ above is

$$
R \Longleftarrow \sum_{n \geq 0} c ? n \cdot d ! n \cdot R+\sum_{n<0} c ? n \cdot c !(n+1) \cdot R
$$

This may be taken to be a description in a pure process algebra where we assume that for each channel name $c$ and for every data-value $v$, in this case every integer, there are atomic actions $c ? v$ and $c ! v$.

There are two disadvantages in this approach. The first is that descriptions which are in some intuitive sense finite are translated into processes which are inherently infinite, at least if the domain of possible values, $\mathrm{Val}$, is infinite; it is necessary to have in the underlying pure process algebra a summation operator $\Sigma_{I}$ where $I$ has the same cardinality as the value domain. Such process algebras are difficult to use. For example the standard algorithms and verification tools, see e.g. [CPS89], do not apply and equational reasoning is difficult since any proof system based on this approach is of necessity infinitary. The second disadvantage is that with such translations uniformities which exist in the object description disappear in the translation. For example the subsequent behaviour of $Q$ above after the reception of an input $v$ is described functionally by the term $\lambda x$.if $x \geq 0$ then $d ! x . Q$ else $c !(x+1)$. $Q$. This uniform treatment of inputs is not apparent in the translation, $R$. Although the notion of uniformity is difficult to define precisely, it should play a central role in proving properties of message passing systems. The object of this paper is to develop a semantic theory of message-passing processes which takes advantage of this uniformity. In particular our semantic theory will apply directly to the syntax of message-passing processes and will not be mediated by a translation into an infinitary language. As a result the associated proof systems will be in some sense finitary.

Such theories already exist for value-passing processes. In [HeI93] a fullyabstract denotational model is presented while in [Hen91] a sound finitary proof system is given which is also complete for recursion free processes. However all of this work is with respect to a particular behavioural equivalence called testing equivalence, [Hen88]. Here we wish to consider an alternative and much finer behavioural equivalence, bisimulation equivalence from [Mil89]. The main result of the paper is a series of sound and complete proof systems, with respect to a range of bisimulation based equivalences, for a recursion free message-passing process algebra.

For most of the paper we restrict our attention to a very simple language which consists essentially of a notation for the empty process, nil, a choice operator + and action prefixing; other operators such as forms of parallel or restriction can easily be accommodated. However, when actions take the form $c ? x$ and $c ! e$, in order for the language to be of interest we also need to be able to test data and 
branch on the consequences of the test. Syntactically this could be represented by an if $b$ then ... else ... construction, where $b$ is a boolean expression, but instead we use the simpler notation of guarded commands, $b \rightarrow t$. Thus

$$
c ? x .(x=0 \rightarrow d ! 0 . n i l+x>0 \rightarrow d ! 1 . n i l)
$$

is a process which inputs a value on $c$ and outputs 0 on $d$ if the input is 0 and 1 if it is greater than 0 and does nothing otherwise. In order to reason about these kinds of processes it is necessary, in general, to reason about data expressions. So following [Hen91] we design a proof system whose judgements are guarded equations of the form

$$
b \triangleright t=u
$$

where $b$ is a boolean expression and $t, u$ are process terms that may contain free data variables. Semantically this should be read as "under any evaluation of free data variables that satisfies $b, t$ is semantically equivalent to $u$ ". The completeness of the proof system is thus relative to that for the data domain involved. Moreover rather than getting embroiled in the details of an actual proof system for data expressions we simply assume the existence of some all powerful mechanism for answering arbitrary questions about data. On the one hand this enables us to concentrate on the behaviour of processes and on the other it reflects what would be a reasonable implementation strategy for a proof system based on our results; the main proof system would be based on the proof rules whose applicability is determined by the structure of processes and this main system would periodically call auxiliary proof systems to establish facts about data expressions. A simple example of a proof rule from the main system is

$$
\frac{b \triangleright t_{i}=u_{i} \quad i=1,2}{b \triangleright t_{1}+t_{2}=u_{1}+u_{2}}
$$

while

$$
\frac{b \models e=e^{\prime}, \quad b \triangleright t=u}{b \triangleright c ! e . t=c ! e^{\prime} . u}
$$

is a rule which depends on a call to an auxiliary proof system concerned with the data domain; this is expressed in rather abstract terms, one of the antecedents referring to the semantics of the expressions $b, e$ and $e^{\prime}$; as we shall see $b \models e=e^{\prime}$ is true if the intended meaning of the boolean $b$ always implies the intended meaning of $e$ equals that of $e^{\prime}$. Of course the reasoning about processes can not be completely divorced from the reasoning about data and an example of where they interact is the cut rule

$$
\begin{array}{ccc}
b \models b_{1} \vee b_{2}, & b_{1} \triangleright t=u \quad b_{2} \triangleright t=u \\
b \triangleright t=u
\end{array}
$$

This enables a proof to be developed by case analysis on the data.

The soundness of such a proof system depends on having a semantic equivalence for processes and as we have already stated in this paper we are interested in bisimulation-like semantics. As a starting point we use strong bisimulation, [Mil89], but as has been pointed out in [MPW92, HeL92] there are at least two natural generalisations of this equivalence to message-passing processes. The first, called early strong bisimulation equivalence, is based on the ability of processes to perform actions of the form $c ? v$ and $c ! v$ while the second is based on the slightly 
more abstract actions $c$ ? and $c ! e$. Thus the processes

$$
c ? x . \operatorname{even}(x) \rightarrow P+c ? x . \operatorname{odd}(x) \rightarrow P
$$

and

$$
c ? x . P+c ? x . n i l
$$

are identified by the early version of the equivalence but are differentiated in the late case because the $c$ ? move from the first to the abstraction

$$
\lambda x . \operatorname{even}(x) \rightarrow P
$$

can not be matched by a corresponding $c$ ? move from the second. Each of these generalisations of strong bisimulation equivalence has a corresponding "weak" version in which internal moves are abstracted. Thus in all we have four reasonable semantic equivalences and for each of these we present a corresponding proof system. In the strong cases the difference between early and late is simply the addition of an axiom, or more correctly an axiom schema, adapted from that used in [PaS93] for the $\pi$-calculus. On the other hand the weak version of both equivalences can be obtained by adding to the corresponding proof system the standard $\tau$-laws from [Mil89].

The judgements of the proof systems involve open process terms, i.e. terms in which data variables need to be instantiated before any operational significance can be associated with them, but the observational equivalences are only defined on closed terms. Therefore in order to even express the soundness and completeness of the proof systems we need to generalise these equivalences to open terms. For each of these semantic equivalences, $\simeq$, we design a proof system with the property that

$$
b \triangleright t=u \text { if and only if } t \rho \simeq u \rho \text { for every evaluation } \rho \text { satisfying } b
$$

As usual establishing soundness is straightforward but completeness requires: some ingenuity. Here we use the approach of [HeL92] and introduce symbolic versions of each of the semantic equivalences which are defined directly on open terms. These are expressed in terms of families of relations over open terms parameterised on boolean expressions and we show that, for each semantic equivalence $\simeq$ we consider,

$$
t \simeq^{b} u \text { if and only if } t \rho \simeq u \rho \text { for every evaluation } \rho \text { satisfying } b
$$

Thus soundness and completeness of the proof systems can be established relative to the symbolic semantic relations $\simeq^{b}$. Using this approach the completeness theorems in particular now become "symbolic versions" of the standard completeness theorems of [Mi189], although the details are somewhat more complicated.

We now give a brief outline of the content of the remainder of the paper. In the next section we define the simple language, give it a concrete operational semantics and define (early) strong bisimulation. This is followed by a discussion of the proof system for proving processes bisimilar. We state the soundness theorem for the system and indicate the difficulty in proving completeness. In the following section, Section 3, we define the symbolic semantics and the associated symbolic bisimulation equivalence and prove that it captures precisely the concrete bisimulation equivalence over processes. We then use this result to show the completeness of the proof system.

In Section 4 we repeat these results for weak bisimulation equivalence where again it is necessary to develop an appropriate definition of weak symbolic equiv- 
alence. The following section outlines corresponding results for a late operational semantics and considers both the strong and weak cases. We end by discussing briefly how to extend these results to other language constructs. We believe that a suitable form of Unique Fixpoint Induction can also be elaborated which will lead to a very useful and powerful proof system for recursively defined processes. However this we leave for future work.

\subsection{Related Work}

We end this section with a brief discussion of related work. As stated previously the approach we have taken is based on that of [Hen91] where a sound and complete proof system for testing equivalence is developed. Here we tackle various bisimulation based equivalences and an essential ingredient of the completeness theorems is the notion of symbolic bisimulation equivalence. This has already been used in [HeL92] to develop an algorithm for checking whether two messagepassing processes are equivalent and in [HeL95] for developing a proof system to verify that such processes satisfy properties described by formulae from a first-order modal logic.

The more standard approach to message-passing processes is to translate them into "pure processes" as outlined at the beginning of this section [Mil89, HoR86]. Indeed in [Bur91] a front-end for the Concurrency Workbench is described which translates message-passing processes from a language such as ours into "pure processes" which can be accepted by the Concurrency Workbench and various examples treated using this approach may be found in [Wal89]. However these approaches require the set of values to be finite and even using the boolean value space of two elements leads to an exponential blow-up in the size of descriptions. We hope that with our approach at least some of this complexity can be avoided. In [Lin93] an extension of the $P A M$ verification system, [Lin91], based on our results, is described. It offers much the same functionality as the the original $P A M$ except that message-passing process algebras can be defined and the proof elaboration scheme is more flexible.

In [GrP90] a very general language for describing message-passing, based on $A C P$, is described and in [GrP91] a proof theory is given. Although these goals are quite similar to ours their approach is very different. A modular algebraic specification language is used to describe data domains and the description of processes is such that it may be viewed as consisting of another module. They continue to view message-passing processes as universally quantified versions of "pure processes", the quantification being over the domain of messages, but they bring to bear the general framework of algebraic specifications in order to handle proof theoretically this quantification. Nevertheless it would be interesting to compare the two approaches.

Recently, proof systems for late and early strong bisimulation equivalences over the $\pi$-calculus, [MPW92], have been given in [PaS93]. Indeed it is from this paper we have borrowed our axiom for the early version of bisimulation equivalence. At one level the $\pi$-calculus may be viewed as a particular instance of a message-passing calculus, where the data-type of messages is the very simple one of channel names. Viewed in this manner our proof systems could be adapted for the $\pi$-calculus and, because of the simplicity of the domain of messages, our proof rules involving the semantic domain of messages would be very simple. But certain uses of channels, in particular their use with the restriction operator to generate private names, means that in fact the $\pi$-calculus is strictly more powerful than our 
notion of a message-passing calculus specialised to the case where the messages are channel names. However this extra complication is adequately provided for in the proof systems of [PaS93]; these achieve much of their power from the blurring of variables and constants which occurs in the $\pi$-calculus.

\section{A Simple Language}

The language we consider can be given by the following BNF grammar

$$
\begin{aligned}
& t \quad:=\text { nil } \mid \text { a.t }|t+t| b \rightarrow t \\
& \alpha::=\tau|c ? x| c ! e
\end{aligned}
$$

where $b$ is a boolean expression, $e$ data expression, $c$ is a channel name and $x$ a data variable. So this syntax assumes a predefined set of channel names, Chan, ranged over by $c$ and a set of data variables, $D V a r$, ranged over by $x, y, \ldots$ More importantly it also assumes a language for data expressions $D E x p$, ranged over by $e, e^{\prime}, \ldots$ and a similar language $B E x p$, ranged over by $b$, for boolean expressions with the usual set of operators $\vee, \wedge, \neg, \ldots$. At the very least we assume that $D E x p$ contains the set of data variables $D V a r$ and also a set of data values $V a l$ and for every pair of data expressions $e, e^{\prime}$ we assume that $e=e^{\prime}$ is a boolean expression. We also assume that all free variables in boolean expressions are data variables. Apart from this we do not worry about the expressive power of these languages although the results on symbolic bisimulations require that the language for boolean expressions is very powerful; sufficiently expressive to characterise arbitrary collections of evaluations.

An evaluation, $\rho$ is a mapping from $D V a r$ to $V a l$ and we use the standard notation $\rho\{v / x\}$ to denote the evaluation which differs from $\rho$ only in that it maps $x$ to $v$. An application of $\rho$ to a data expression $e$, denoted $\rho(e)$, always yields a value from $\mathrm{Val}$ and similarly for boolean expressions; $\rho(b)$ is either true or false. Thus we assume that evaluation of data and boolean expressions always terminate and our approach is to work modulo these evaluations. We also assume that these evaluations satisfy standard properties; each expression $e$ has associated with it a set of variables $f v(e)$ and, for example, if $\rho$ and $\rho^{\prime}$ agree on $f v(e)$ then $\rho(e)=\rho^{\prime}(e)$. If an expression $e$ has no variables, it is closed, then $\rho(e)$ is independent of $\rho$ and we use $\llbracket e \rrbracket$ to denote its value. Similarly with boolean expressions. We will use the suggestive notation $b \models b^{\prime}$ to indicate that for every evaluation $\rho$ if $\rho(b)$ is true then so is $\rho\left(b^{\prime}\right)$. Of course we could equally well say that $b \rightarrow b^{\prime}$ is a logical theorem but our notation emphasises the fact that we wish to work modulo the semantics of expressions. In line with this notation we use $\rho \models b$ to indicate that $\rho(b)=t r u e$. We will also write $b=b^{\prime}$ for $b \models b^{\prime}$ and $b^{\prime} \models b$.

We will also refer to substitutions, and assume that they satisfy the expected properties; we use $e\left[e^{\prime} / x\right]$ to denote the result of substituting $e^{\prime}$ for all occurrences of $x$ in $e$. More generally a substitution $\sigma$ is a mapping from data variables to expressions and we use $e \sigma$ to denote the result of applying $\sigma$ to the expression $e$.

Returning to the process language above, the prefix $c ? x$ binds the occurrence of $x$ in the sub-term $t$ of $c$ ?x.t and we have as usual the sets of free variables $f v(u)$ and bound variables $b v(u)$ of a term $u$; of course these depend in general on the variables in the data and boolean expressions contained in $u$. This leads to the standard definition of $\alpha$-conversion, $\equiv_{\alpha}$, over terms and of substitution, $t[e / x]$ denoting the result of substituting all free occurrences of $x$ in $t$ by $e$, and this relies on the definition of substitution in data expressions. A term is closed if it 


$$
\begin{aligned}
& \tau . p \stackrel{\tau}{\longrightarrow} p \\
& \text { c!e.p } \stackrel{c ! v}{\longrightarrow} p \\
& c ? x . t \stackrel{c ? v}{\longrightarrow} t[v / x] \\
& p \stackrel{a}{\rightarrow} e p^{\prime} \\
& \text { implies } \\
& \text { where } \llbracket e \rrbracket=v \\
& c \in \text { Chan, } v \in \text { Val } \\
& p \stackrel{a}{\longrightarrow} p^{\prime}, \llbracket b \rrbracket=\text { true } \quad \text { implies } \\
& p+q \stackrel{a}{\rightarrow} e p^{\prime} \\
& q+p \stackrel{a}{\longrightarrow} e p^{\prime} \\
& b \rightarrow p \stackrel{a}{\longrightarrow} p^{\prime}
\end{aligned}
$$

Fig. 1. (Early) Operational semantics.

$$
\begin{array}{ll}
\text { S1 } & X+n i l=X \\
\text { S2 } & X+X=X \\
\text { S3 } & X+Y=Y+X \\
\text { S4 } & (X+Y)+Z=X+(Y+Z)
\end{array}
$$

Fig. 2. The axioms $\mathscr{A}_{1}$.

contains no free variables and these we refer to as processes, ranged over by $p, q, \ldots$. Throughout the paper open terms refer to terms that may contain free occurrences of data variables, but no process variables. Open terms are ranged over by $t, u, \ldots$; we give the following precedence to the operators (in decreasing order) : $\rightarrow .+$

The standard operational semantics of this language is given in Fig. 1. It consists of a set of binary relations, $\stackrel{a}{\longrightarrow}$, between processes, where $a$ ranges over the set $A c t=\{\tau, c ? v, c ! v \mid v \in V a l\}$. In [MPW92, HeL92] this is referred to as the early operational semantics as when input terms such as $c ? x . p$ perform an action the value received is immediately bound to the variable $x$. In Section 5 we will see a slightly different way of organising input actions where this binding is delayed.

A symmetric relation $R$ between closed terms is a strong bisimulation if it satisfies: $(p, q) \in R$ implies that for every $a \in A c t$

$$
\text { whenever } p \stackrel{a}{\longrightarrow} p^{\prime} \text { then there exists } q \stackrel{a}{\longrightarrow} e q^{\prime} \text { and }\left(p^{\prime}, q^{\prime}\right) \in R
$$

where a ranges over $\{\tau, c ? v, c ! v \mid v \in V a l\}$. We use $\sim_{e}$ to denote the largest (early) strong bisimulation. This relation generalizes naturally to open terms by letting $t \sim_{e} u$ iff $t \rho \sim_{e} u \rho$ for any $\rho$. We then have

Proposition 2.1. $\sim_{e}$ is preserved by every operator in the language.

By transition induction it can be easily shown that $\alpha$-equivalent processes have the same transitions (up to $\alpha$-equivalence):

Lemma 2.1. If $p \equiv_{\alpha} q$ and $p \stackrel{a}{\rightarrow} p^{\prime}$ then $q \stackrel{a}{\rightarrow} q^{\prime}$ for some $q^{\prime} \equiv_{\alpha} p^{\prime}$.

From this lemma it follows that $\alpha$-equivalent processes are bisimilar:

Proposition 2.2. If $p \equiv_{\alpha} q$ then $p \sim_{e} q$.

We now consider a proof system for deriving statements about $p \sim_{e} q$. In general we will need to consider open terms because in order to prove a statement such as $c ? x . t=c ? x . u$ it is necessary to relate the open terms $t$ and $u$. Also because we allow testing of data we will need to establish statements relative to a boolean expression $b$. Thus the judgements are guarded equations of the form 


$$
\begin{aligned}
& \text { EQUIV } \quad \frac{b \triangleright t=u}{\text { true } \triangleright t=t} \quad \frac{b \triangleright t=u, u=v}{b \triangleright u=t} \quad \frac{b \triangleright t=v}{b \triangleright t} \\
& \text { EQN } \frac{}{\text { true } \triangleright t \sigma=u \sigma} \quad t=u \text { is an axiom } \\
& \text { CONGR } \frac{b \triangleright t_{i}=u_{i} \quad i=1,2}{b \triangleright t_{1}+t_{2}=u_{1}+u_{2}} \\
& \alpha \text {-CONV } \quad \overline{\text { true } \triangleright c ? x . t=c ? y \cdot t[y / x]} \quad y \notin f v(t) \\
& \text { INPUT } \frac{b \triangleright t=u}{b \triangleright c ? x . t=c ? x . u} \quad x \notin f v(b) \\
& \text { OUTPUT } \frac{b \models e=e^{\prime}, \quad b \triangleright t=u}{b \triangleright c ! e . t=c ! e^{\prime} \cdot u} \\
& \mathrm{TAU} \quad \begin{aligned}
b \triangleright t & =u \\
\hline b \triangleright \tau . t & =\tau . u
\end{aligned} \\
& \text { GUARD } \frac{b \wedge b^{\prime} \triangleright t=u \quad b \wedge \neg b^{\prime} \triangleright n i l=u}{b \triangleright b^{\prime} \rightarrow t=u} \\
& \text { CUT } \quad \frac{b \models b_{1} \vee b_{2}, \quad b_{1} \triangleright t=u \quad b_{2} \triangleright t=u}{b \triangleright t=u} \\
& \text { ABSURD } \text { false } \triangleright t=u
\end{aligned}
$$

Fig. 3. The inference rules.

$$
b \triangleright t=u
$$

where $b$, the guard, is a boolean expression. For brevity we usually write $t=u$ for true $\triangleright t=u$.

The basis for the proof system are the standard set of equations for strong bisimulation equivalence over CCS, [Mil89], given in Fig. 2. The rules for the proof system are given in Fig. 3; in the rule EQN $\sigma$ is any mapping from process variables to process terms. Note that reference is made to the semantics of data expressions in the OUTPUT rule, for establishing identities of the form $c ! e . t=c ! e^{\prime} . u$, and in the CUT rule, which is used to perform proofs by case analysis. The rule GUARD also uses a case analysis; an identity of the form $b \rightarrow t=u$ may be established by considering two cases, one when $b$ is true and the other when it is false. This means that the development of a proof in this system, specifically the application of the OUTPUT and CUT rules, requires the establishment of facts about the data domain. These are the only two rules which rely on such facts but they can be used to derive other useful rules of a similar nature such as

$$
\text { CONSEQUENCE } \frac{b=b^{\prime}, b^{\prime} \triangleright t=u}{b \triangleright t=u}
$$

The side condition, $x \notin f v(b)$, in the rule INPUT is essential, as otherwise it would not be sound. It could be used to prove 


$$
x=1 \triangleright c ? x . c ! 1 . \text { nil }=c ? x . c ! x . \text { nil }
$$

because

$$
x=1 \triangleright c ! 1 . n i l=c ! x . n i l .
$$

With this side condition it is sound but not sufficiently powerful to derive all true identities between early bisimilar processes. For example the two processes discussed in the introduction

$$
c ? x . \text { even }(x) \rightarrow P+c ? x . \operatorname{odd}(x) \rightarrow P \text { and } n c ? x . P+c ? x . n i l
$$

are strong bisimulation equivalent but can not be proved equivalent using this restricted rule.

To overcome this problem we adapt the axiom used in [PaS93] to characterise early strong bisimulation equivalence in the $\pi$-calculus to obtain the axiom schema:

$$
\text { EA } c ? x . t+c ? x \cdot u=c ? x . t+c ? x \cdot u+c ? x(b \rightarrow t+\neg b \rightarrow u) .
$$

This is an absorption law. The process term $c$ ?x.t $+c$ ?x.u can absorb the term $c ? x(b \rightarrow t+\neg b \rightarrow u)$ for any boolean expression $b$.

Let us write $\vdash_{1} b \triangleright t=u$ to mean that $b \triangleright t=u$ can be derived from these equations using the rules in Fig. 3.

The soundness of $t_{1}$ is given by the following proposition:

Proposition 2.3. If $\vdash_{1} b \triangleright t=u$ and $\rho \models b$ then $t \rho \sim_{e} u \rho$

Proof. The proof is by induction on the derivation of $b \vdash_{1} t=u$ and a case analysis on the last rule used.

The converse to this is also true but the proof is far from straightforward. The problem arises because $\sim_{e}$ is only defined on closed terms whereas the proof system manipulates open terms. So there is no straightforward way to use structural induction on terms. Instead we develop a symbolic version of bisimulation equivalence for open terms which captures the standard bisimulation equivalence on all instantiations and then prove completeness with respect to this symbolic version.

Symbolic bisimulations are the topic of the next section and we finish the present section with some useful facts about the proof system, mainly concerning the guard construct:

\section{Proposition 2.4.}

1. $\vdash_{1} b \rightarrow b^{\prime} \rightarrow t=b \wedge b^{\prime} \rightarrow t$

2. $\vdash_{1} t=t+b \rightarrow t$

3. $b \models b^{\prime}$ implies $\vdash_{1} b \triangleright t=b^{\prime} \rightarrow t$

4. $\vdash_{1} b \wedge b^{\prime} \triangleright t=u$ implies $\vdash_{1} b \triangleright b^{\prime} \rightarrow t=b^{\prime} \rightarrow u$

5. $\vdash_{1} b \rightarrow(t+u)=b \rightarrow t+b \rightarrow u$

6. $\vdash_{1} b \rightarrow u+b^{\prime} \rightarrow u=b \vee b^{\prime} \rightarrow u$

7. if $f v(b) \cap b v(\alpha)=\emptyset$ then $\vdash_{1} b \rightarrow \alpha . t=b \rightarrow \alpha .(b \rightarrow t)$

Proof. As examples we prove two of these statements.

- 2. Because true $\models b \wedge \neg b$, by the cut rule it is sufficient to prove the two statements 


$$
\vdash_{1} b \triangleright t=t+b \rightarrow t \text { and } \vdash_{1} \neg b \triangleright t=t+b \rightarrow t
$$

The first is derived by an application of the equation S2 and $\vdash_{1} b \triangleright t=b \rightarrow t$. This in turn is established by the GUARD rule applied to

$$
\vdash_{1} b \wedge b \triangleright t=t \text { and } b \wedge \neg b \triangleright t=n i l
$$

which are simple consequences of EQUIV and ABSURD respectively. The second statement above is derived by an application of S1 and

$$
\vdash_{1} \neg b \triangleright b \rightarrow t=\text { nil }
$$

This in turn is an easy consequence of the GUARD rule.

- 7. Two applications of GUARD and two of ABSURD reduce this to

$$
\vdash_{1} b \triangleright \alpha . t=\alpha .(b \rightarrow t)
$$

The proof now depends on the nature of $\alpha$. For example if it is $c ? x$ we know that $x \notin f v(b)$ and therefore by INPUT it can be reduced to $\vdash_{1} b \triangleright t=b \rightarrow t$ which in turn follows from 3 .

If $\alpha$ is $\tau$ or $c ! e$ the derivation is even more straighforward.

As an illustration of the usefulness of this proposition we use it to drive a generalisation of the axiom EA:

Lemma 2.2. If $b_{1} \vee b_{2}=b$ and $b_{1} \wedge b_{2}=$ false then

$$
\vdash_{1} b \triangleright c ? x . t+c ? x . u=c ? x . t+c ? x . u+c ? x(b \rightarrow t+\neg b \rightarrow u)
$$

Proof. By $\alpha$-conversion we may assume $x \notin f v(b)$. Since $b_{1} \wedge\left(b_{2} \vee \neg b\right)=$ false and $b_{1} \vee\left(b_{2} \vee \neg b\right)=$ true, applying EA we have

$$
\vdash_{1} c ? x . t+c ? x . u=c ? x . t+c ? x . u+c ? x .\left(b_{1} \rightarrow t+b_{2} \vee \neg b \rightarrow u\right)
$$

Hence, using Proposition 2.4,

$$
\begin{aligned}
\vdash_{1} b & \triangleright c ? x . t+c ? x . u \\
& =c ? x . t+c ? x . u+c ? x . b \rightarrow\left(b_{1} \rightarrow t+b_{2} \vee \neg b \rightarrow u\right) \\
& =c ? x . t+c ? x . u+c ? x .\left(b \wedge b_{1} \rightarrow t+b \wedge\left(b_{2} \vee \neg b\right) \rightarrow u\right) \\
& =c ? x . t+c ? x . u+c ? x .\left(b_{1} \rightarrow t+b_{2} \rightarrow u\right)
\end{aligned}
$$

\section{Symbolic Bisimulations}

The reader is refered to [HeL92] for motivation and discussion on symbolic bisimulations. Here we adapt the definitions, which were originally given for symbolic graphs, to our language.

The abstract or symbolic transition relations are defined to be the least set of relations which satisfy the rules in Fig. 4 . They take the form of relations $\stackrel{b, \alpha}{\longrightarrow}$ between open terms, where $b$ is a boolean expression and $\alpha$ is a prefix, i.e. it has one of the forms $\tau, c ? x$ or $c ! e$. Intuitively $b$ acts like a guard: it enables the move when it is true. The bound variable used in the symbolic input transitions is not significant as the following lemma emphasises:

\section{Lemma 3.1.}

1. If $t \stackrel{b, \alpha}{\longrightarrow} t^{\prime}$ then $f v(b) \subseteq f v(t), b v(\alpha) \cap f v(t)=\emptyset$ and $f v\left(t^{\prime}\right) \subseteq f v(t) \cup b v(\alpha)$. 


$$
\begin{array}{lll}
\alpha . t \stackrel{\text { true, } \alpha}{\longrightarrow} t & \alpha \in\{\tau, c ! e \mid c \in \text { Chan,e Exp }\} \\
c ? x . t \stackrel{\text { true,c?y }}{\longrightarrow} t[y / x] & y \notin f v(c ? x . t) \\
t \stackrel{b^{\prime}, \alpha}{\longrightarrow} t^{\prime} & \text { implies } & b \rightarrow t \stackrel{b \wedge b^{\prime}, \alpha}{\longrightarrow} t^{\prime} \\
t \stackrel{b, \alpha}{\longrightarrow} t^{\prime} & \text { implies } & t+u \stackrel{b, \alpha}{\longrightarrow} t^{\prime} \\
& & u+t \stackrel{b, \alpha}{\longrightarrow} t^{\prime}
\end{array}
$$

Fig. 4. Symbolic operational semantics.

2. If $t \stackrel{b, c ? x}{\longrightarrow} t^{\prime}$ then $t \stackrel{b, c ? y}{\longrightarrow} t^{\prime}[y / x]$ for any $y \notin f v(t)$.

Proof. By transition induction.

The symbolic actions can be related to the concrete actions in the following manner:

\section{Lemma 3.2.}

1. If $t \rho \stackrel{\tau}{\longrightarrow} \rho$ then there exist $b, t^{\prime}$ s.t. $\rho \models b, p \equiv_{\alpha} t^{\prime} \rho$ and $t \stackrel{b, \tau}{\longrightarrow} t^{\prime}$.

2. If $t \stackrel{b, \tau}{\longrightarrow} t^{\prime}, \rho \models b$ then $t \rho \stackrel{\tau}{\longrightarrow} p$ for some $p \equiv_{\alpha} t^{\prime} \rho$.

\section{Lemma 3.3.}

1. If $t \rho \stackrel{c ! v}{\longrightarrow} p$ then there exist $b, e, t^{\prime}$ s.t. $\rho \models b, \rho(e)=v, p \equiv_{\alpha} t^{\prime} \rho$ and $t \stackrel{b, c ! e}{\longrightarrow} t^{\prime}$.

2. If $t \stackrel{b, c ! e}{\longrightarrow} t^{\prime}, \rho \models b$ then $t \rho \stackrel{c ! v}{\longrightarrow} p$ for some $p \equiv_{\alpha} t^{\prime} \rho$ where $v=\rho(e)$.

\section{Lemma 3.4 .}

1. If $t \rho \stackrel{c ? v}{\longrightarrow} p, x \notin f v(t)$ then there exist $b, t^{\prime}$ s.t. $\rho \models b, p \equiv_{\alpha} t^{\prime} \rho\{v / x\}$ and $t \stackrel{b, c ? x}{\rightarrow} t^{\prime}$

2. If $t \stackrel{b, c ? x}{\longrightarrow} t^{\prime}, \rho \models b$ then for any $v \in$ Val $t \rho \stackrel{c ? v}{\longrightarrow}_{e} p$ for some $p \equiv_{\alpha} t^{\prime} \rho\{v / x\}$.

Proof. These lemmas can be proved by induction on the derivation of transitions. As an example we prove Lemma 3.4.

1. Apply induction on why $t \rho \stackrel{c ? v}{\longrightarrow} p$.

- $(c ? y \cdot u) \rho \stackrel{c ? v}{\longrightarrow} u \rho\{v / y\}, x \notin f v(c ? y . u)$. Then $c ? y . u \stackrel{\text { true }, c ? x}{\longrightarrow} u[x / y]$ and $u \rho\{v / y\} \equiv_{\alpha}$ $u[x / y] \rho\{v / x\}$.

- $(b \rightarrow u) \rho \stackrel{c ? v}{\longrightarrow} p$ is because $\rho=b$ and $u \rho \stackrel{c ? v}{\longrightarrow} p$. By induction there exist $b^{\prime}, t^{\prime}$ s.t. $\rho \models b^{\prime}, p \equiv_{\alpha} t^{\prime} \rho\{v / x\}$ and $u \stackrel{b^{\prime}, c ? x}{\longrightarrow} t^{\prime}$. Hence $b \rightarrow u \stackrel{b \wedge b^{\prime}, c ? x}{\longrightarrow} t^{\prime}$ and $\rho \vDash b \wedge b^{\prime}$.

- $u \rho+q \stackrel{c ? v}{\longrightarrow} p$ is because $u \rho \stackrel{c ? v}{\longrightarrow} p$. Similar.

2. Apply induction on why $t \stackrel{b, c ? x}{\longrightarrow} t^{\prime}$.

- $c ? y . u \stackrel{\text { true,c?x }}{\longrightarrow} u[x / y], \quad x \notin f v(c ? y . u)$. We have $(c ? y . u) \rho \stackrel{c ? v}{\longrightarrow} u[v / x] \rho \equiv_{\alpha}$ $u[x / y] \rho\{v / x\}$.

- $b \rightarrow u \stackrel{b \wedge b^{\prime}, c ? x}{\longrightarrow} t^{\prime}$ is because $u \stackrel{b^{\prime}, c ? x}{\longrightarrow} t^{\prime}$. Since $\rho \models b \wedge b^{\prime}, \rho \models b^{\prime}$. By induction $u \rho \stackrel{c ? v}{\longrightarrow}_{e} p \equiv_{\alpha} t^{\prime} \rho$. Hence $(b \rightarrow u) \rho \stackrel{c ?_{v}}{\longrightarrow} p$. 


\section{- $t+u \stackrel{b, c ? x}{\longrightarrow} t^{\prime}$ is because $t \stackrel{b, c ? x}{\longrightarrow} t^{\prime}$. Similar.}

Based on these symbolic actions we can define $\mathscr{E} \mathscr{S} \mathscr{B}$, (early symbolic bisimulations), which, for reasons explained in [HeL92], must be parameterised on boolean expressions. A finite set of boolean expressions $B$ is called a $b$-partition if $\bigvee B=b$. Let $\mathbf{S}=\left\{S^{b} \mid b \in B \operatorname{Exp}\right\}$ be a family of relations over terms, indexed by boolean expressions. Then $\mathscr{E} \mathscr{S} \mathscr{B}(\mathbf{S})$ is the family of symmetric relations defined by:

$(t, u) \in \mathscr{E} \mathscr{P} \mathscr{B}(\mathbf{S})^{b}$ if whenever $t \stackrel{b_{1}, \alpha}{\longrightarrow} t^{\prime}$ with $b v(\alpha) \cap f v(b, t, u)=\emptyset$, there is a $b \wedge b_{1}$-partition $B$ with the property that for each $b^{\prime} \in B$ there exists a $u \stackrel{b_{2}, \alpha^{\prime}}{\longrightarrow} u^{\prime}$ such that $b^{\prime}=b_{2}$ and

1. if $\alpha=c ! e$ then $\alpha^{\prime}=c ! e^{\prime}, b^{\prime} \models e=e^{\prime}$ and $\left(t^{\prime}, u^{\prime}\right) \in S^{b^{\prime}}$

2. otherwise $\alpha=\alpha^{\prime}$ and $\left(t^{\prime}, u^{\prime}\right) \in S^{b^{\prime}}$

Definition 3.1. (Symbolic Bisimulations) $\mathbf{S}$ is an (early) strong symbolic bisimulation if $\mathbf{S} \subseteq \mathscr{E} \mathscr{S} \mathscr{B}(\mathbf{S})$, where $\subseteq$ is point-wise inclusion.

Let $\sim_{\mathbf{E}}=\left\{\sim_{E}^{b}\right\}$ be the largest (early) strong symbolic bisimulation.

The interest in symbolic bisimulations lies in the fact they are defined with respect to the abstract operational semantics, which for finite terms can be represented as a finite transition graph; in contrast the standard "concrete" bisimulations are defined over infinite transitions graphs, at least if the set of values is infinite. In [HeL92] we give an algorithm for checking for this symbolic equivalence. Here we use it to show completeness of the proof systems. First we relate symbolic and concrete bisimulation equivalence.

Theorem 3.1. (Soundness and completeness of $\sim_{E}$ )

$t \sim_{E}^{b} u$ iff $t \rho \sim_{e} u \rho$ for every evaluation $\rho$ such that $\rho \models b$.

Proof. (Outline) The proof follows the corresponding result in [HeL92], Theorem 6.5 ; it consists in establishing a relationship between symbolic bisimulations and concrete ones. If $\mathbf{S}=\left\{S^{b}\right\}$ is a strong symbolic bisimulation let

$$
R_{\mathbf{S}}=\left\{(t \rho, u \rho) \mid \exists b, \rho \models b \text { and }(t, u) \in S^{b}\right\} .
$$

Soundness follows immediately if we can prove that $R_{\mathrm{S}}$ is a bisimulation. Conversely if $R$ is a strong bisimulation let

$$
S^{b}=\{(t, u) \mid \rho \models b \text { implies }(t \rho, u \rho) \in R\}
$$

for any boolean expression $b$. Completeness follows, as in [HeL92], if we can show that $\mathbf{S}=\left\{S^{b}\right\}$ is a symbolic bisimulation.

The proof of these two subsidiary results depends on the relationship between the abstract actions to the concrete actions given in Lemmas 3.2, 3.3 and 3.4. The details are similar to Theorem 4.2 .

With this theorem we can now return to the proof system and show its completeness by proving

$$
t \sim_{E}^{b} u \text { implies } \vdash_{1} b \triangleright t=u
$$

This provides the converse to Proposition 2.3. The proof of (*) follows the standard proof of the corresponding "concrete" result, as given in [Mil89], except that now we work at the symbolic level. It is by induction on the size of terms which is defined as follows: 
1. $\mid$ nil $\mid=0$

2. $|t+u|=\max \{|t|,|u|\}$

3. $|b \rightarrow t|=|t|$

4. $|\alpha . t|=1+|t|$

We also need the notion of normal form:

Definition 3.2. $\mathrm{t}$ is a normal form, or in normal form, if it has the form $\Sigma_{i} b_{i} \rightarrow \alpha_{i} . t_{i}$ and each $t_{i}$ is in normal form.

Lemma 3.5. For every term $t$ there exists a normal form $t^{\prime}$ such that $f v(t)=$ $f v\left(t^{\prime}\right),|t|=\left|t^{\prime}\right|$ and $\vdash_{1} t=t^{\prime}$.

Proof. By structural induction on terms using the elementary facts about the proof system given in Proposition 2.4.

The following generalisation of the axiom EA will be useful in the exposition of the completeness proof.

Proposition 3.1. For any finite non-empty collection of booleans $\left\{b_{i} \mid 1 \leq i \leq n\right\}$, such that $\bigvee_{i \in I} b_{i}=b$ and $b_{i} \wedge b_{j}=$ false for $i \neq j$,

$$
\vdash_{1} b \triangleright \sum_{1 \leq i \leq n} c ? x . t_{i}=\sum_{1 \leq i \leq n} c ? x . t_{i}+c ? x . \sum_{1 \leq i \leq n} b_{i} \rightarrow t_{i}
$$

Proof. By induction on $n$. The base case is trivial. Now assume the result for $n-1$ with $n>1$ and let $b_{i}^{\prime}=b_{i}$ for $1 \leq i<n-1$ and $b_{n-1}^{\prime}=b_{n-1} \vee b_{n}$. Then $\bigvee_{1 \leq i \leq n-1} b_{i}^{\prime}=b, b_{i}^{\prime} \wedge b_{j}^{\prime}=$ false for $i \neq j$, and $\left(\bigvee_{1 \leq i \leq n-1} b_{i}\right) \wedge b_{i}^{\prime}=b_{i}$ for $1 \leq i \leq n-1$. We have

$$
\begin{aligned}
& \vdash_{1} b \triangleright \quad \sum_{1 \leq i \leq n} c ? x . t_{i} \\
& =\quad \sum_{1 \leq i \leq n-1} c ? x . t_{i}+c ? x . t_{n} \\
& =\quad \sum_{1 \leq i \leq n-1}^{\leq} c ? x . t_{i}+c ? x \cdot\left(\sum_{1 \leq i \leq n-1} b_{i}^{\prime} \rightarrow t_{i}\right)+c ? x . t_{n}
\end{aligned}
$$

$$
\begin{array}{ll}
\text { Prop 2.4 } & \sum_{1 \leq i \leq n-1} c ? x \cdot t_{i}+c ? x \cdot\left(\sum_{1 \leq i \leq n-1} b_{i}^{\prime} \rightarrow t_{i}\right)+c ? x . t_{n}+ \\
& c ? x \cdot\left(\sum_{1 \leq i \leq n-1} b_{i} \rightarrow t_{i}+b_{n} \rightarrow t_{n}\right) \\
= & \sum_{1 \leq i \leq n-1} c ? x \cdot t_{i}+c ? x \cdot\left(\sum_{1 \leq i \leq n-1} b_{i}^{\prime} \rightarrow t_{i}\right)+c ? x . t_{n}+ \\
& c ? x \cdot\left(\sum_{1 \leq i \leq n} b_{i} \rightarrow t_{i}\right) \\
= & \sum_{1 \leq i \leq n} c ? x \cdot t_{i}+c ? x \cdot\left(\sum_{1 \leq i \leq n} b_{i} \rightarrow t_{i}\right)
\end{array}
$$


Theorem 3.2. (Completeness of $\vdash_{1}$ ) $t \sim_{E}^{b} u$ implies $\vdash_{1} b \triangleright t=u$

Proof. By induction on the joint size of $t$ and $u$. We may assume that both are normal forms, $t \equiv \Sigma_{i \in I} c_{i} \rightarrow \alpha_{i} . t_{i}$ and $u \equiv \Sigma_{j \in J} d_{j} \rightarrow \beta_{j} . u_{j}$. Call a prefix of type $\gamma \in\{\tau, c !, c$ ? $\mid c \in$ Chan $\}$ if it has the form $\tau, c ! e, c ? x$, respectively. Let $I_{\gamma}=\left\{i \in I \mid \alpha_{i}\right.$ has type $\left.\gamma\right\}, J_{\gamma}=\left\{j \in J \mid \beta_{j}\right.$ has type $\left.\gamma\right\}$ and also $t_{\gamma}, u_{\gamma}$ denote $\Sigma_{i \in I_{\gamma}} c_{i} \rightarrow \alpha_{i} . t_{i}, \Sigma_{j \in J_{\gamma}} d_{j} \rightarrow \beta_{j} . u_{j}$ respectively. We show $\vdash_{1} b \triangleright t_{\gamma}=u_{\gamma}$ for each type $\gamma$. Clearly $t_{\gamma} \sim_{E}^{b} u_{\gamma}$. Because of $\alpha$-conversion we may assume that each input prefix in $t_{c}$ ? and $u_{c}$ ? uses the same variable $x \notin f v(t, u, b)$. We examine the cases $\gamma=\tau$ and $\gamma=c$ ? here and leave the case $\gamma=c$ ! to the reader.

- (Case $\gamma=\tau$ ).

By symmetry we need only to show

$$
\vdash_{1} b \triangleright u_{\tau}+c_{i} \rightarrow \tau . t_{i}=u_{\tau}
$$

for each $i \in I_{\tau}$. Note that $\vdash_{1} b \wedge \neg c_{i} \triangleright c_{i} \rightarrow \tau . t_{i}=$ nil so by CUT it is sufficient to show

$$
\vdash_{1} b \wedge c_{i} \triangleright u_{\tau}+c_{i} \rightarrow \tau . t_{i}=u_{\tau}
$$

Since $\vdash_{1} b \wedge c_{i} \triangleright c_{i} \rightarrow \tau . t_{i}=t_{i}$ this may be further simplified to

$$
\vdash_{1} b \wedge c_{i} \triangleright u_{\tau}+\tau . t_{i}=u_{\tau}
$$

Now $t_{\tau} \stackrel{c_{i}, \tau}{\longrightarrow} t_{i}$. So there exists a $b \wedge c_{i}$-partition $B$ such that for each $b^{\prime} \in B$ there is $u_{\tau} \stackrel{d_{j}, \tau}{\longrightarrow} u_{j}$ such that $b^{\prime}=d_{j}$ and $t_{i} \sim{ }_{E}^{b^{\prime}} u_{j}$. By induction $\vdash_{1} b^{\prime} \triangleright t_{i}=u_{j}$. By TAU $\vdash_{1} b^{\prime} \triangleright \tau . t_{i}=\tau . u_{j}$. Since $b^{\prime}=d_{j}$ we have $\vdash_{1} b^{\prime} \triangleright t_{i}=d_{j} \rightarrow \tau . u_{j}$ and by $\mathrm{S} 2 \vdash_{1} b^{\prime} \triangleright u_{\tau} \tau . t_{i}=u_{\tau}$. This is true for each $b^{\prime}$ in $B$ and therefore an application of CUT gives the required

$$
\vdash_{1} b \wedge c_{i} \triangleright u_{\tau}+\tau . t_{i}=u_{\tau}
$$

- (Case $\gamma=c$ ?).

As in the previous case it is sufficient to prove that

$$
\vdash_{1} b \wedge c_{i} \triangleright u_{c} ?+c ? x . t_{i}=u_{c} ?
$$

for an arbitrary $i \in I_{c}$.

For each $L \subseteq J_{c}$, let $d_{L}=\left(\bigwedge_{j \in L} d_{j}\right) \wedge\left(\bigwedge_{j^{\prime} \in J_{c} \text { ? }}-L d_{j^{\prime}}\right)$. Then $\bigvee_{L \subseteq J_{c ?}} d_{L}=$ true and $d_{L} \wedge d_{L^{\prime}}=$ false for $L \neq L^{\prime}$. Using Proposition 2.4 we can derive

$$
\vdash_{1} u_{c ?}=\Sigma_{L \subseteq J_{c} ?}\left(d_{L} \rightarrow \Sigma_{j \in L} \mathcal{c} ? x . u_{j}\right)
$$

By CUT, (1) can be reduced to showing that, for each $L$,

$$
\vdash_{1} b \wedge c_{i} \wedge d_{L} \triangleright u_{c}=u_{c}+c ? x . t_{i}
$$

which, by (2), can be further reduced to

$$
\vdash_{1} b \wedge c_{i} \wedge d_{L} \triangleright \Sigma_{j \in L} c ? x . u_{j}=\Sigma_{j \in L} c ? x . u_{j}+c ? x . t_{i}
$$

We show how to derive this for an arbitrary $L$. Note that by $\alpha$-conversion we can assume that $x$ does not occur free in $b \wedge c_{i} \wedge d_{L}$.

Since $u_{c}$ ? $\sim_{E}^{b \wedge c_{i} \wedge d_{L}} t_{c}$ ? and $t_{c} ? \stackrel{c_{i}, c ? x}{\longrightarrow} t_{i}$, there exists a $b \wedge c_{i} \wedge d_{L}$-partition $B$ such that for each $b^{\prime} \in B, u_{c}$ ? $\stackrel{d_{j_{b^{\prime}}}, c ? x}{\longrightarrow} u_{j_{b^{\prime}}}$ for some $j_{b^{\prime}}$ with $b^{\prime} \models d_{j_{b^{\prime}}}$ and $t_{i} \sim_{E}^{b^{\prime}} u_{j_{b^{\prime}}}$. 
Without loss of generality we may assume the booleans in $B$ are mutual disjoint, i.e. $b^{\prime} \wedge b^{\prime \prime}=$ false for any $b^{\prime}, b^{\prime \prime} \in B$.

By induction, $\vdash_{1} b^{\prime} \triangleright t_{i}=u_{j_{b^{\prime}}}$ or, equivalently, $\vdash_{1} b^{\prime} \rightarrow t_{i}=b^{\prime} \rightarrow u_{j_{b^{\prime}}}$. So

$$
\begin{aligned}
\vdash_{1} \Sigma_{b^{\prime} \in B} b^{\prime} \rightarrow u_{j_{b^{\prime}}} & =\Sigma_{b^{\prime} \in B} b^{\prime} \rightarrow t_{i} \\
& =b \wedge c_{i} \wedge d_{L} \rightarrow t_{i}
\end{aligned}
$$

Hence $\vdash_{1} b \wedge c_{i} \wedge d_{L} \triangleright \Sigma_{b^{\prime} \in B} b^{\prime} \rightarrow u_{j_{b^{\prime}}}=t_{i}$. Now, because $x \notin f v\left(b \wedge c_{i} \wedge d_{L}\right)$, we can apply INPUT to obtain

$$
\vdash_{1} b \wedge c_{i} \wedge d_{L} \triangleright c ? x . \Sigma_{b^{\prime} \in B} b^{\prime} \rightarrow u_{j_{b^{\prime}}}=c ? x . t_{i}
$$

This identity can in fact be strengthened to

$$
\vdash_{1} b \wedge c_{i} \wedge d_{L} \triangleright c ? x . \Sigma_{b^{\prime} \in B^{\prime}} b^{\prime} \rightarrow u_{j_{b^{\prime}}}=c ? x . t_{i}
$$

where $B^{\prime}=\{b \in B \mid b \neq$ false $\}$. The advantage of using $B^{\prime}$ in place of $B$ is that $b^{\prime} \in B^{\prime}$ implies that $j_{b^{\prime}} \in L$ : we know $b^{\prime} \models d_{L} \wedge d_{j_{b^{\prime}}}$ and so $d_{L} \wedge d_{j_{b^{\prime}}} \neq$ false which because of the construction of $d_{L}$ immediately implies $j_{b^{\prime}} \in L$.

Therefore

$$
\begin{aligned}
& \vdash_{1} \quad b \wedge c_{i} \wedge d_{L} \triangleright \Sigma_{j \in L} c ? x . u_{j} \\
& \stackrel{\text { s2 }}{=} \quad \Sigma_{j \in L} c ? x . u_{j}+\Sigma_{b^{\prime} \in B^{\prime}} c ? x . u_{j_{b^{\prime}}} \\
& \text { Prop. } 3.1 \quad \Sigma_{j \in L} c ? x . u_{j}+\Sigma_{b^{\prime} \in B^{\prime}} c ? x . u_{j_{b^{\prime}}}+\Sigma_{b^{\prime} \in B^{\prime}} c ? x . b^{\prime} \rightarrow u_{j_{b^{\prime}}} \\
& \stackrel{(5)}{=} \quad \Sigma_{j \in L} c ? x . u_{j}+\Sigma_{b^{\prime} \in B^{\prime}} c ? x \cdot u_{j_{b^{\prime}}}+c ? x . t_{i} \\
& \stackrel{S 2}{=} \quad \Sigma_{j \in L} c ? x . u_{j}+c ? x . t_{i}
\end{aligned}
$$

This is the required (3) above.

Our proposed axiom schema EA is very general since it allows us to introduce an arbitrary boolean expression $b$ into a proof. In previous versions of this work, [?], we had a different approach; instead of the axiom schema EA we used the following rule schema:

$$
\text { E-INPUT } \frac{b \triangleright \sum_{i \in I} \tau . t_{i}=\sum_{j \in J} \tau . u_{j}}{b \triangleright \sum_{i \in I} c ? x . t_{i}=\sum_{j \in J} c ? x . u_{j}} \quad x \notin f v(b) .
$$

This also is quite general but at least its application only depends on the structure of the terms in the proof being elaborated and is at least somewhat schematic.

Note that the use of $\tau$ in this rule is essential. For example the rule

$$
\frac{b \triangleright \sum_{i \in I} t_{i}=\sum_{j \in J} u_{j}}{b \triangleright \sum_{i \in I} c ? x . t_{i}=\sum_{j \in J} c ? x . u_{j}} \quad x \notin f v(b)
$$

is unsound. For example since $(p+q)+r \sim_{e} p+(q+r)$ for all processes $p, q, r$ we could use this rule to derive

$$
c ? x \cdot(p+q)+c ? x . r \sim_{e} c ? x \cdot p+c ? x \cdot(q+r)
$$

for which there are obvious counterexamples.

With the help of GUARD and CUT, EA can be easily derived from EINPUT. Hence the proof system obtained by replacing the axiom schema EA with E-INPUT is also complete. A direct completeness proof is also possible: The only change is to the last case examined in the proof of Theorem 3.2, when $\gamma$ is $c$ ?. The details can be found in the proof of Theorem 4.3. 


$$
\begin{array}{ll}
\text { T1 } & \alpha . \tau . X=\alpha . X \\
\text { T2 } & X+\tau . X=\tau . X \\
\text { T3 } & \alpha .(X+\tau . Y)+\alpha . Y=\alpha .(X+\tau . Y)
\end{array}
$$

Fig. 5. The axioms $\mathscr{A}_{2}$.

\section{Weak Bisimulation Equivalence}

In this section we outline how to extend the results of the previous two sections to so-called weak bisimulations.

The concrete double arrows $\stackrel{a}{\Longrightarrow}$, where $a \in\{\varepsilon, \tau, c ? v, c ! v\}$, are defined as the least relations between closed terms generated by the following rules

- $p \stackrel{\varepsilon}{=} p$.

- $p \stackrel{a}{\longrightarrow} q$ implies $p \stackrel{a}{\longrightarrow} q$.

- $p \stackrel{\tau}{\longrightarrow} \stackrel{a}{\longrightarrow}_{e} q$ implies $p \stackrel{a}{=} \stackrel{a}{e}_{e}$.

- $p \stackrel{a}{\Longrightarrow} \stackrel{\tau}{\longrightarrow}_{e} q$ implies $p \stackrel{a}{\Longrightarrow} q$.

The weak version of Lemma 2.1 holds:

Lemma 4.1. If $p \equiv_{\alpha} q$ and $p \stackrel{a}{\Longrightarrow} p^{\prime}$ then $q \stackrel{a}{\Longrightarrow} q^{\prime}$ for some $q^{\prime} \equiv_{\alpha} p^{\prime}$.

Let $\hat{a}$ to be $\varepsilon$ when $a=\tau$, and $a$ otherwise. The early weak bisimulation is then defined as usual (for closed terms):

Definition 4.1. $R$ is an early weak bisimulation if $(p, q) \in R$ implies

- if $p \stackrel{a}{\longrightarrow} p^{\prime}$ then $q \stackrel{\hat{a}}{\longrightarrow} q^{\prime}$ for some $q^{\prime}$ such that $\left(p^{\prime}, q^{\prime}\right) \in R$.

- if $q \stackrel{a}{\longrightarrow} q^{\prime}$ then $p \stackrel{\hat{a}}{\longrightarrow} p^{\prime}$ for some $p^{\prime}$ such that $\left(p^{\prime}, q^{\prime}\right) \in R$.

Let $\approx_{e}$ be the largest early weak bisimulation.

The aim of this section is to extend the proof system of Section 2 to weak bisimulation equivalence. However it is well-known that $\approx_{e}$ is not preserved by + and so we have to work with the modified relation:

Definition 4.2. Two closed terms $p, q$ are early observation congruent, written $p \simeq_{e} q$, if for all $a \in\{\tau, c ? v, c ! v\}$

- Whenever $p \stackrel{a}{\longrightarrow} e p^{\prime}$ then $q \stackrel{a}{\Longrightarrow} q^{\prime}$ for some $q^{\prime}$ such that $p^{\prime} \approx_{e} q^{\prime}$.

- Whenever $q \stackrel{a}{\longrightarrow} q^{\prime}$ then $p \stackrel{a}{\Longrightarrow} p^{\prime}$ for some $p^{\prime}$ such that $p^{\prime} \approx_{e} q^{\prime}$.

As usual $\simeq_{e}$ is the largest congruence relation contained in $\approx_{e}$. This relation can be generalized to open terms by letting $t \simeq_{e} u$ iff $t \rho \simeq_{e} u \rho$ for any $\rho$. We then have the standard result

Proposition 4.1. $\simeq_{e}$ is preserved by all the operators in the language.

To give a sound and complete proof system for this relation, it is sufficient to add to the proof system the equations $\mathscr{A}_{2}$ given in Fig. 5. Let us use $\vdash_{2} b \triangleright t=u$ to denote that $b \triangleright t=u$ can be derived from the proof system using the axioms $\mathscr{A}_{2}$, and of course $\mathscr{A}_{1}$. For the sake of variety in this section we work with the version of the proof system which uses the proof rule E-INPUT rahter than the 
axiom schema EA; This will provide an opportunity of outlining a proof of a completeness theorem involving E-INPUT, in contrast to the completeness proof of the previous section which uses EA.

The main result of this section is

Theorem 4.1. (Soundness and completeness of $\vdash_{2}$ )

$\vdash_{2} b \triangleright t=u$ if and only if $t \rho \simeq_{e} u \rho$ for every $\rho$ such that $\rho \models b$.

The soundness is straightforward, by induction on the length of the derivation of $b \triangleright t=u$. The strategy for proving completeness is the same as in the strong case. We first develop a symbolic version of weak bisimulation and relate it to the concrete version. We then prove completeness with respect to this symbolic version of bisimulation congruence.

First we define the symbolic double arrows as the least relations between open terms which satisfy:

- $t \stackrel{\text { true, },}{\Longrightarrow} t$.

- $t \stackrel{b, \alpha}{\longrightarrow} u$ implies $t \stackrel{b, \alpha}{\Longrightarrow} u$.

- $t \stackrel{b, \tau}{\longrightarrow} \stackrel{b^{\prime}, \alpha}{\Longrightarrow}=$ implies $t \stackrel{b \wedge b^{\prime}, \alpha}{\Longrightarrow} u$.

- $t \stackrel{b, \alpha}{\Longrightarrow}{\stackrel{b^{\prime}, \tau}{\longrightarrow}}^{\longrightarrow} u$ implies $t \stackrel{b \wedge b^{\prime}, \alpha}{\Longrightarrow}{ }_{E} u$.

It will be necessary to use a slight variation on these in the late case and therefore we use the index $E$ to indicate that these are early weak symbolic moves.

Concerning bound variables we now have

Lemma 4.2. If $t \stackrel{b, c ? x}{\Longrightarrow} t^{\prime}$ then $f v(b) \subseteq f v(t) \cup\{x\}$ and $x \notin f v(t)$.

That is, in a double input transition the input variable can appear in the guard.

The two versions of double arrows can also be related as in the case of single arrows.

\section{Lemma 4.3.}

1. If $t \rho \stackrel{\tau}{\Longrightarrow}_{e} p$ then there exist $b$, $t^{\prime}$ s.t. $\rho \models b, p \equiv_{\alpha} t^{\prime} \rho$ and $t \stackrel{b, \tau}{=} t_{E}^{\prime}$.

2. If $t \stackrel{b, \tau}{\Longrightarrow} t^{\prime}, \rho \models b$ then $t \rho \stackrel{\tau}{\Longrightarrow}_{e} p$ for some $p \equiv_{\alpha} t^{\prime} \rho$.

\section{Lemma 4.4.}

1. If $t \rho \stackrel{\varepsilon}{\Longrightarrow} p$ then there exist $b, t^{\prime}$ s.t. $\rho \models b, p \equiv_{\alpha} t^{\prime} \rho$ and $t \stackrel{b, \varepsilon}{\Longrightarrow} t_{E} t^{\prime}$.

2. If $t \stackrel{b, \varepsilon}{\Longrightarrow} E t^{\prime}, \rho \models b$ then $t \rho \stackrel{\varepsilon}{\Longrightarrow} p$ for some $p \equiv_{\alpha} t^{\prime} \rho$.

\section{Lemma 4.5 .}

1. If $t \rho \stackrel{c ! v}{\Longrightarrow} p$ then there exist $b, e, t^{\prime}$ s.t. $\rho \models b, \rho(e)=v, p \equiv_{\alpha} t^{\prime} \rho$ and $t \stackrel{b, c ! e}{\Longrightarrow} t_{E}^{\prime}$.

2. If $t \stackrel{b, c l e}{\Longrightarrow} t^{\prime}, \rho \models b$ then $t \rho \stackrel{c ! v}{\Longrightarrow} p$ for some $p \equiv_{\alpha} t^{\prime} \rho$ where $v=\rho(e)$.

\section{Lemma 4.6.}

1. If $t \rho \stackrel{c ? v}{\Longrightarrow} p, x \notin f v(t)$ then there exist $b, t^{\prime}$ s.t. $f v(b) \subseteq f v(t) \cup\{x\}, \rho\{v / x\}=$ $b, p \equiv_{\alpha} t^{\prime} \rho\{v / x\}$ and $t \stackrel{b, c ? x}{\Longrightarrow} t^{\prime}$. 
2. If $t \stackrel{b, c ? x}{\Longrightarrow} t^{\prime}, \rho\{v / x\} \models b$ then $t \rho \stackrel{c ? v}{\Longrightarrow} e$ for some $p \equiv_{\alpha} t^{\prime} \rho\{v / x\}$.

Proof. The proofs of these four lemmas are quite similar and as an example we prove Lemma 4.6.

1. We will prove a slightly stronger statement:

If $t \rho \stackrel{c ? v}{\Longrightarrow} p, x \notin f v(t)$ then there exist $b, b^{\prime}, t^{\prime}$ s.t. $f v(b) \subseteq f v(t), \rho \models b$, $f v\left(b^{\prime}\right) \subseteq f v(t) \cup\{x\}, \rho\{v / x\} \vDash b, p \equiv_{\alpha} t^{\prime} \rho\{v / x\}$ and $t \stackrel{b \wedge b^{\prime}, c ? x}{\Longrightarrow} t_{E}^{\prime}$.

The proof is by induction on why $t \rho \stackrel{c ? v}{\Longrightarrow} p$.

- $t \rho \stackrel{c ? v}{\longrightarrow} p$. Immediate from Lemma 3.4 and the definition of $\Longrightarrow_{E}$.

- $t \rho \stackrel{\tau}{\longrightarrow} e q \stackrel{c ? v}{\Longrightarrow} e p$. By Lemma 3.4 there exist $b^{\prime \prime}, t^{\prime \prime}$ s.t. $\rho \models b^{\prime \prime}, q \equiv_{\alpha}$ $t^{\prime \prime} \rho$ and $t \stackrel{b^{\prime \prime}, \tau}{\longrightarrow} t^{\prime \prime}$. By Lemma $3.1 f v\left(b^{\prime \prime}\right) \subseteq f v(t), f v\left(t^{\prime \prime}\right) \subseteq f v(t)$. Apply Lemma 4.1 we get $t^{\prime \prime} \rho \stackrel{c ? v}{\Longrightarrow} p^{\prime} \equiv_{\alpha} p$. So by induction $\exists b^{\prime \prime \prime}, b^{\prime}$, $t^{\prime}$ s.t. $x \notin f v\left(b^{\prime \prime \prime}\right), \rho \models b^{\prime \prime \prime}, \rho\{v / x\} \vDash b^{\prime}, p^{\prime} \equiv_{\alpha} t^{\prime} \rho\{v / x\}$ and $t^{\prime \prime} \stackrel{b^{\prime \prime \prime} \wedge b^{\prime}, c ? x}{\Longrightarrow} t_{E}^{\prime}$. Let $b=b^{\prime \prime} \wedge b^{\prime \prime \prime}$ then $x \notin f v(b), \rho \models b$ and $t \stackrel{b \wedge b^{\prime}, c ? x}{\Longrightarrow} t_{E}^{\prime}$.

- $t \rho \stackrel{c ?_{v}}{\longrightarrow} q \stackrel{\tau}{\longrightarrow} \rho$. Similar to the previous case.

2. By induction on why $t \stackrel{b, c ? x}{\Longrightarrow} t_{E}^{\prime}$.

- $t \stackrel{b, c ? x}{\longrightarrow} t^{\prime}$. Straightforward from Lemma 3.4 and the definition of $\Longrightarrow_{e}$.

- $t \stackrel{b^{\prime}, \tau}{\longrightarrow} u \stackrel{b^{\prime \prime}, c ? x}{\Longrightarrow} t_{E}^{\prime}$ with $b \equiv b^{\prime} \wedge b^{\prime \prime}$. Then $x \notin f v\left(b^{\prime}\right)$, so $\rho \models b^{\prime}$. By Lemma 3.2, $t \rho \stackrel{\tau}{\longrightarrow}_{e} q \equiv_{\alpha} u \rho$. By induction $u \rho \stackrel{c ? c}{\longrightarrow} p^{\prime} \equiv_{\alpha} t^{\prime} \rho\{v / x\}$. By Lemma 4.1 $q \stackrel{c ? v}{\Longrightarrow} p \equiv_{\alpha} p^{\prime}$. By the definition of $\Longrightarrow_{e}, t \rho \stackrel{c ? v}{\Longrightarrow} p \equiv_{\alpha} t^{\prime} \rho\{v / x\}$.

- $t \stackrel{b^{\prime}, c ? x}{\Longrightarrow} u \stackrel{b^{\prime \prime}, \tau}{\longrightarrow} t^{\prime}$ with $b \equiv b^{\prime} \wedge b^{\prime \prime}$. By induction $t \rho \stackrel{c ? v}{\Longrightarrow} q \equiv_{\alpha} u \rho\{v / x\}$. By Lemma $3.2 u \rho\{v / x\} \stackrel{\tau}{\longrightarrow} p^{\prime} \equiv_{\alpha} t^{\prime} \rho\{v / x\}$. By Lemma $2.1 q \stackrel{\tau}{\longrightarrow} p p \equiv_{\alpha} p^{\prime}$. Therefore $t \rho \stackrel{c ? v}{\Longrightarrow} p \equiv_{\alpha} t^{\prime} \rho\{v / x\}$.

Now let $\mathbf{S}=\left\{S^{b} \mid b \in \operatorname{Exp}\right\}$ be a family of relations over terms indexed by boolean expressions. Then $\mathscr{E} \mathscr{W} \mathscr{B}(\mathbf{S})$ is the family of symmetric relations defined by:

$(t, u) \in \mathscr{E} \mathscr{W} \mathscr{B}(\mathbf{S})^{b}$ if whenever $t \stackrel{b_{1}, \alpha}{\longrightarrow} t^{\prime}$ with $b v(\alpha) \cap f v(b, t, u)=\emptyset$, then there is a $b \wedge b_{1}$-partition $B$ such that for each $b^{\prime} \in B$ there exists a $u \stackrel{b_{2}, \hat{\alpha}^{\prime}}{\Rightarrow} u_{E}^{\prime}$ such that $b^{\prime}=b_{2}$ and

1. if $\alpha \equiv c ! e$ then $\alpha^{\prime} \equiv c ! e^{\prime}, b^{\prime} \models e=e^{\prime}$ and $\left(t^{\prime}, u^{\prime}\right) \in S^{b^{\prime}}$

2. otherwise $\alpha \equiv \alpha^{\prime}$ and $\left(t^{\prime}, u^{\prime}\right) \in S^{b^{\prime}}$

Definition 4.3. (Weak Symbolic Bisimulations) $\mathrm{S}$ is a weak symbolic bisimulation if $\mathbf{S} \subseteq \mathscr{E} \mathscr{W} \mathscr{B}(\mathbf{S})$

Let $\approx_{\mathbf{E}}=\left\{\approx_{E}^{b}\right\}$ be the largest (early) weak symbolic bisimulation.

Again we have to modify $\approx_{E}$ so that it is preserved by + : 
Definition 4.4. Two terms $t, u$ are symbolic observation congruent with respect to $b$, written $t \simeq_{E}^{b} u$, if whenever $t \stackrel{b_{1}, \alpha}{\longrightarrow} t^{\prime}$ with $b v(\alpha) \cap f v(b, t, u)=\emptyset$, then there is a $b \wedge b_{1}$-partition $B$ with the following property: for each $b^{\prime} \in B$ there exists a $u \stackrel{b_{2}, \alpha^{\prime}}{\Longrightarrow} u^{\prime}$ such that $b^{\prime} \models b_{2}$ and

1. if $\alpha \equiv c$ !e then $\alpha^{\prime} \equiv c ! e^{\prime}, b^{\prime} \models e=e^{\prime}$ and $t^{\prime} \approx_{E}^{b^{\prime}} u^{\prime}$

2. otherwise $\alpha \equiv \alpha^{\prime}$ and $t^{\prime} \approx_{E}^{b^{\prime}} u^{\prime}$ and symmetrically for $u$.

Note that in this definition it is still essential to use partitions when matching moves. For example

$$
\tau . p \simeq_{E}^{\text {true }} b \rightarrow \tau . p+\neg b \rightarrow \tau . \tau . p
$$

but the symbolic move $\tau . p \stackrel{\text { true } \tau}{\longrightarrow} p$ can not be matched properly by a single symbolic move from the right hand side.

The two versions of weak bisimulation equivalence/congruence can be related as in the case of strong bisimulation.

Theorem 4.2. (Soundness and completeness of $\approx_{E}$ and $\simeq_{E}$ )

1. $t \approx_{E}^{b} u$, where $f v(b) \subseteq f v(t, u)$, if and only if $t \rho \approx_{e} u \rho$ for every $\rho$ such that $\rho \vDash b$.

2. $t \simeq_{E}^{b} u$, where $f v(b) \subseteq f v(t, u)$, if and only if $t \rho \simeq_{e} u \rho$ for every $\rho$ such that $\rho \vDash b$.

Proof. We only prove 1 . The proof of 2 is similar.

$(\Longrightarrow)$ Let

$$
R=\left\{(t \rho, u \rho) \mid \exists b, f v(b) \subseteq f v(t, u), \rho \models b \text { and } t \approx_{E}^{b} u\right\}
$$

We show $R$ is a weak early bisimulation.

Suppose $(t \rho, u \rho) \in R$, i.e. $\exists b, f v(b) \subseteq f v(t, u), \rho \models b$ and $t \approx_{E}^{b} u$. Let $t \rho \stackrel{a}{\longrightarrow} e$. we must find a matching transition from $u \rho$. There are three cases to consider.

- $t \rho \stackrel{\tau}{\longrightarrow} p$. By Lemma 3.2(1), $\exists b_{1}$ and $t$ s.t. $\rho \models b_{1}, p \equiv_{\alpha} t^{\prime} \rho$ and $t \stackrel{b_{1}, \tau}{\longrightarrow} t^{\prime}$. So $\exists b \wedge b_{1}$-partition $B$ with the properties guaranteed by Definition 4.3. Since $\rho \models b \wedge b_{1}$ and $\vee B=b \wedge b_{1}, \exists b^{\prime} \in B$ s.t. $\rho \models b^{\prime}$. Let $u \stackrel{b_{2}, \varepsilon}{\Longrightarrow} u^{\prime}$ be the symbolic transition associated with this $b^{\prime}$. Then $\rho \models b_{2}$ and $t^{\prime} \approx_{E}^{b^{\prime}} u^{\prime}$. By Lemma $4.4(2) u \rho \stackrel{\varepsilon}{\Rightarrow} \rightleftharpoons_{e} q \equiv_{\alpha} u^{\prime} \rho$. Moreover $\left(t^{\prime} \rho, u^{\prime} \rho\right) \in R$ by the definition of $R$. Hence $(p, q) \in R$.

- $t \rho \stackrel{c ? v}{\longrightarrow} p$. By Lemma 3.4(1), $\exists b_{1}, x$, and $t^{\prime}$ s.t. $x \notin f v(t), \rho \models b_{1}, \quad p \equiv_{\alpha}$ $t^{\prime} \rho\{v / x\}$ and $t \stackrel{b_{1}, c ? x}{\longrightarrow} t^{\prime}$. We may assume $x \notin f v(u)$. So $\exists b \wedge b_{1}$-partition $B$ with the properties guaranteed by Definition 4.3. Since $\rho=b \wedge b_{1}$ and $x \notin$ $f v\left(b \wedge b_{1}\right), \rho\{v / x\} \vDash=b \wedge b_{1}$. So $\exists b^{\prime} \in B, \rho\{v / x\} \models b^{\prime}$. Let $u \stackrel{b_{2}, c ? x}{\Longrightarrow} u^{\prime}$ be the symbolic transition associate with this $b^{\prime}$, then $b^{\prime} \models b_{2}$ and $t^{\prime} \approx_{E}^{b^{\prime}} u^{\prime}$. Since $\rho\{v / x\} \models b_{2}$, by Lemma 4.6(2), $u \rho \stackrel{c ? v}{\Longrightarrow} q \equiv_{\alpha} u^{\prime} \rho\{v / x\}$. Since $\rho\{v / x\} \models b^{\prime}$ and $t^{\prime} \approx_{E}^{b^{\prime}} u^{\prime},\left(t^{\prime} \rho, u^{\prime} \rho\right) \in R$. Hence $(p, q) \in R$. 
- $t \rho \stackrel{c ! v}{\longrightarrow} p$. Similar.

$(\Longleftrightarrow)$ We show the boolean-indexed family of relations $\mathbf{S}=\left\{S^{b} \mid b \in B E x p\right\}$ defined by

$$
S^{b}=\left\{(t, u) \mid f v(b) \subseteq f v(t, u), \rho \models b \text { implies } t \rho \approx_{e} u \rho\right\}
$$

is a weak early symbolic bisimulation.

Suppose $(t, u) \in S^{b}$ and let $t \stackrel{b_{1}, \alpha}{\longrightarrow} t^{\prime}$. consider three cases:

- $t \stackrel{b_{1}, \tau}{\longrightarrow} t^{\prime}$. We need to construct a $b \wedge b_{1}$-partition $B$ with the required properties.

To this end we number all $\varepsilon$-transitions from $u$ thus

$$
u \stackrel{b_{2}^{i}, \varepsilon}{\Longrightarrow} u^{i}, \quad 0<i \leq k
$$

For each $i$, let $b_{i}^{\prime \prime}$ be a boolean with the following properties:

$$
\begin{aligned}
& f v\left(b_{i}^{\prime \prime}\right) \subseteq f v(t, u) \\
& \rho \models b_{i}^{\prime \prime} \text { iff } t^{\prime} \rho \approx_{e} u^{i} \rho
\end{aligned}
$$

Let $b_{i}^{\prime}=b \wedge b_{1} \wedge b_{2}^{i} \wedge b_{i}^{\prime \prime}$ and $B=\left\{b_{i}^{\prime} \mid 0<i \leq k\right\}$. We first show that $B$ is a $b \wedge b_{1}$-partition, i.e. $\bigvee B=b \wedge b_{1}$. By construction $\bigvee B \models b \wedge b_{1}$. Now we show $b \wedge b_{1} \models \bigvee B$.

Let $\rho \models b \wedge b_{1}$. Then $t \rho \approx_{e} u \rho$. By Lemma 3.2(2), $t \rho \stackrel{\tau}{\longrightarrow} p p \equiv_{\alpha} t^{\prime} \rho$. So there exists $u \rho \stackrel{\varepsilon}{=} \rho q \approx_{e} p$. By Lemma 4.4(1), $\exists b_{2}^{i}, u^{i}$ s.t. $\rho \models b_{2}^{i}, q \equiv_{\alpha}$ $u^{i} \rho$ and $u \stackrel{b_{2}^{i}, \varepsilon}{\Longrightarrow} u^{i}$. Then $t^{\prime} \rho \approx_{e} u^{i} \rho$. By the definition of $b_{i}^{\prime \prime}, \rho \models b_{i}^{\prime \prime}$. Therefore $\rho \models b_{i}^{\prime}$. Hence $\rho \models \bigvee B$.

It is easy to see that $B$ has the required property: for any $b_{i}^{\prime} \in B, u \stackrel{b_{2}^{i}, \varepsilon}{\Longrightarrow} u^{i}$ with $b_{i}^{\prime} \models b_{i}^{\prime \prime}$. Moreover $\left(t^{\prime}, u^{i}\right) \in S^{b_{i}^{\prime}}$ by the definition of $b_{i}^{\prime \prime}$ and $\mathbf{S}$.

- $t \stackrel{b_{1}, c ? x}{\longrightarrow} t^{\prime}$. We may assume $x \notin f v(u)$. Similar to the previous case we number all weak $c$ ? $x$-transitions from $u$ thus

$$
u \stackrel{b_{2}^{i}, c ? x}{\Longrightarrow} u^{i}, \quad 0<i \leq k
$$

For each $i$, let $b_{i}^{\prime \prime}$ be a boolean with the following properties:

$$
\begin{aligned}
& f v\left(b_{i}^{\prime \prime}\right) \subseteq f v(t, u) \cup\{x\} \\
& \text { For any } v, \rho\{v / x\} \vDash b_{i}^{\prime \prime} \text { iff } t^{\prime} \rho\{v / x\} \approx_{e} u^{i} \rho\{v / x\}
\end{aligned}
$$

Let $b_{i}^{\prime}=b \wedge b_{1} \wedge b_{2}^{i} \wedge b_{i}^{\prime \prime}$ and $B=\left\{b_{i}^{\prime} \mid 0<i \leq k\right\}$. First we check that $B$ is a $b \wedge b_{1}$-partition. Again $\bigvee B \models b \wedge b_{1}$ by construction. To see $b \wedge b_{1} \models B$, let $\rho \models b \wedge b_{1}$. Then $t \rho \approx_{e} u \rho$. By Lemma 3.4(1), for any $v \in V a l, t \rho \stackrel{c ? v}{\longrightarrow} e_{e}$ $p \equiv_{\alpha} t^{\prime} \rho\{v / x\}$. So there exists $u \rho \stackrel{c t v}{=} q \approx_{e} p$. By Lemma 4.6(1), $\exists b_{2}^{i}, u^{i}$ s.t. $f v\left(b_{2}^{i}\right) \in f v(u) \cup\{x\}, \rho\{v / x\} \models b_{2}^{i}, q \equiv_{\alpha} u^{i} \rho\{v / x\}$ and $u \stackrel{b_{2}^{i}, c ? x}{\Rightarrow} u_{E}^{i}$. Hence $t^{\prime} \rho\{v / x\} \approx_{e} u^{i} \rho\{v / x\}$. From the definition of $b_{i}^{\prime \prime}, \rho\{v / x\} \models b_{i}^{\prime \prime}$. Furthermore, since $\rho \models b \wedge b_{1}$ and $x \notin f v\left(b \wedge b_{1}\right), \rho\{v / x\} \models b \wedge b_{1}$. Thereofre $\rho\{v / x\} \models b_{i}^{\prime}$. Hence $\rho\{v / x\} \models \vee B$. Since $v$ is arbitrary, $\rho \models B$.

By the construction of $B$ it is easy to see that $B$ has the required porperty: 
For any $b_{i}^{\prime} \in B, u \stackrel{b_{2}^{i}, c ? x}{\Longrightarrow} u^{i}$ and $b_{i}^{\prime} \models b_{2}^{i}$. If $\rho \models b_{i}^{\prime}$ then $\rho \models b_{i}^{\prime \prime}$. Take $v=\rho(x)$, then $\rho\{v / x\}=\rho$, so $t^{\prime} \rho \approx_{e} u^{i} \rho$. Hence $\left(t^{\prime}, u^{i}\right) \in S^{b_{i}^{\prime}}$ by the definition of $B$.

- $t \stackrel{b_{1}, c ! e}{\longrightarrow} t^{\prime}$. Similar.

We now turn our attention to the completeness of the proof system. First we need the following generalisation of the axiom T3:

Lemma 4.7. If $f v(b) \cap b v(\alpha)=\emptyset$ then $\vdash_{2} \alpha .(X+b \rightarrow \tau . Y)=\alpha .(X+b \rightarrow$ $\tau . Y)+b \rightarrow \alpha . Y$

Proof. Since $X=X+b \rightarrow X$ we need only to show

$$
\vdash_{2} b \rightarrow \alpha .(X+b \rightarrow \tau . Y)=b \rightarrow \alpha .(X+b \rightarrow \tau . Y)+b \rightarrow \alpha . Y
$$

which can be derived as follows (using Proposition 2.4):

$$
\begin{aligned}
& \vdash_{2} \quad b \rightarrow \alpha .(X+b \rightarrow \tau . Y) \\
& =b \rightarrow \alpha .(b \rightarrow(X+b \rightarrow \tau . Y)) \\
& =b \rightarrow \alpha .(b \rightarrow(X+\tau . Y)) \\
& =b \rightarrow \alpha .(X+\tau . Y) \\
& =b \rightarrow(\alpha .(X+\tau . Y)+\alpha . Y) \\
& =b \rightarrow \alpha .(X+\tau . Y)+b \rightarrow \alpha . Y \\
& =b \rightarrow \alpha .(X+b \rightarrow \tau . Y)+b \rightarrow \alpha . Y
\end{aligned}
$$

(previous steps reversed)

Completeness of the proof system will follow if we can prove $t \simeq_{E}^{b} u$ implies $\vdash_{2} b \triangleright t=u$. The following two results are essential to this proof; they are symbolic versions of two results which also play an essential role in the completeness proof for "pure" CCS, [Mil89].

Lemma 4.8. (Absorption) If $t \stackrel{b, \alpha}{\Longrightarrow} E t^{\prime}$ with $f v(b) \cap b v(\alpha)=\emptyset$, then $\vdash_{2} t=t+b \rightarrow$ $\alpha . t^{\prime}$.

Proof. By induction on why $t \stackrel{b, \alpha}{\Longrightarrow} E t^{\prime}$.

1. $t \stackrel{b, \alpha}{\longrightarrow} t^{\prime}$.

- $\alpha^{\prime} \cdot t_{1} \stackrel{\text { true, } \alpha}{\longrightarrow} t^{\prime}$ with $\alpha^{\prime} . t_{1} \equiv_{\alpha} \alpha . t^{\prime}$. Use S3.

- $b^{\prime} \rightarrow t_{1} \stackrel{b^{\prime} \wedge b^{\prime \prime}, \alpha}{\longrightarrow} t^{\prime}$ because $t_{1} \stackrel{b^{\prime \prime}, \alpha}{\longrightarrow} t^{\prime}$. By induction $\vdash_{2} t_{1}=t_{1}+b^{\prime \prime} \rightarrow \alpha . t^{\prime}$. So

$$
\begin{aligned}
\vdash_{2} b^{\prime} \rightarrow t_{1} & =b^{\prime} \rightarrow\left(b^{\prime \prime} \rightarrow \alpha \cdot t^{\prime}\right) \\
& =b^{\prime} \rightarrow t_{1}+b^{\prime} \wedge b^{\prime \prime} \rightarrow \alpha . t^{\prime} \text { by Proposition 2.4. }
\end{aligned}
$$

- The other cases are similar.

2. $t \stackrel{b^{\prime}, \alpha}{\longrightarrow} t_{1} \stackrel{b^{\prime \prime}, \tau}{\Longrightarrow} E t^{\prime}$ with $b \equiv b^{\prime} \wedge b^{\prime \prime}$. By induction $\vdash_{2} t_{1}=t_{1}+b^{\prime \prime} \rightarrow \tau \cdot t^{\prime}$ and $\vdash_{2} t=t+b^{\prime} \rightarrow \alpha . t_{1}$. So, since $b v(\alpha) \cap f v(b)=\emptyset$,

$$
\begin{aligned}
\vdash_{2} t & =t+b^{\prime} \rightarrow \alpha .\left(t_{1}+b^{\prime \prime} \rightarrow \tau . t^{\prime}\right) \\
& =t+b^{\prime} \rightarrow\left(\alpha .\left(t_{1}+b^{\prime \prime} \rightarrow \tau . t^{\prime}\right)+b^{\prime \prime} \rightarrow \alpha . t^{\prime}\right) \text { by Lemma } 4.7 \\
& =t+b^{\prime} \rightarrow b^{\prime \prime} \rightarrow \alpha . t^{\prime} \\
& =t+b \rightarrow \alpha . t^{\prime}
\end{aligned}
$$


3. $t \stackrel{b^{\prime}, \tau}{\Longrightarrow} E t_{1} \stackrel{b^{\prime \prime}, \alpha}{\longrightarrow} t^{\prime}$ with $b \equiv b^{\prime} \wedge b^{\prime \prime}$. Similar to the previous case.

Proposition 4.2. $t \approx_{E}^{b} u$ if and only if there is a $b$-partition $B$ such that for all $b^{\prime} \in B, t \simeq_{E}^{b^{\prime}} u$ or $t \simeq_{E}^{b^{\prime}} \tau . u$ or $\tau . t \simeq_{E}^{b^{\prime}} u$

Proof. The "if" part is trivial because of Theorem 4.2. For the "only if" part, by a construction similar to that used in Proposition 4.3, we can assume $t \equiv$ $\Sigma_{i \in I} c_{i} \rightarrow \Sigma_{k \in K_{i}} \alpha_{i k} . t_{i k}, u \equiv \Sigma_{j \in J} d_{j} \rightarrow \Sigma_{l \in L_{j}} \beta_{j l} . u_{j l}$, where $c_{i} \wedge c_{i^{\prime}}=$ false for $i \neq i^{\prime}$, $d_{j} \wedge d_{j^{\prime}}=$ false for $j \neq j^{\prime}, \bigvee_{i \in I} c_{i}=$ true, and $\bigvee_{j \in J} d_{j}=$ true.

Set $B^{\prime}=\left\{b \wedge c_{i} \wedge d_{j} \mid i \in I, j \in J\right\}$. Then $\vee B^{\prime}=b$. Consider an arbitrary $b^{\prime} \equiv b \wedge c_{i} \wedge d_{j} \in B^{\prime}$. Since $b^{\prime} \models b, t \approx_{E}^{b^{\prime}} u$. So for every $t \stackrel{c_{i}, \tau}{\longrightarrow} t_{i k}$, there exists a $b^{\prime}$-partition $B_{i k}$ with the property that for each $b_{1} \in B_{i k}$ there is a $u \stackrel{d_{j}, \hat{\imath}}{\Longrightarrow} u_{E}^{\prime}$ s.t. $t_{k} \approx_{E}^{b_{1}} u^{\prime}$, and for every $u \stackrel{d_{j, \tau}}{\longrightarrow} u_{j l}$ there exists a $b^{\prime}$-partition $B_{j l}$ with the property that for each $b_{2} \in B_{j l}$ there is a $t \stackrel{c_{i}, \hat{t}}{\Longrightarrow} E t^{\prime}$ s.t. $t^{\prime} \approx_{E}^{b_{2}} u_{j l}$.

Let $B^{1}=\left\{\wedge_{k \in K_{i}} b_{k} \mid b_{k} \in B_{i k}\right\}, B^{2}=\left\{\wedge_{l \in L_{j}} b_{l} \mid b_{l} \in B_{j l}\right\}, \quad$ and $B_{b^{\prime}}=$ $\left\{b_{1} \wedge b_{2} \mid b_{1} \in B^{1}, b_{2} \in B^{2}\right\}$. Then $\bigvee B_{b^{\prime}}=b^{\prime}$. Furthermore $B_{b^{\prime}}$ has the property that for each $b^{\prime \prime} \in B_{b^{\prime}}, t \approx_{E}^{b^{\prime \prime}} u$, and whenever $t \stackrel{c_{i}, \tau}{\longrightarrow} t^{\prime}$, there is a $u \stackrel{d_{j}, \hat{\imath}}{\Longrightarrow} u^{\prime} \approx_{E}^{b^{\prime \prime}} t^{\prime}$; whenever $u \stackrel{d_{j}, \tau}{\longrightarrow} u^{\prime}$ there is a $t \stackrel{c_{i}, \hat{\tau}}{\longrightarrow} t^{\prime} \approx_{E}^{b^{\prime \prime}} u^{\prime}$. If for some $t^{\prime} t \stackrel{c_{i}, \tau}{\longrightarrow} t^{\prime} \approx_{E}^{b^{\prime \prime}} u$ then $t \simeq_{E}^{b^{\prime \prime}} \tau . u$; If for some $u^{\prime} u \stackrel{d_{j, t}}{\longrightarrow} u^{\prime} \approx_{E}^{b^{\prime \prime}} t$ then $\tau . t \simeq_{E}^{b^{\prime \prime}} u$. Otherwise we can show $t \simeq_{E}^{b^{\prime \prime}} u$ as follows. Let $t \stackrel{c_{i}, x}{\longrightarrow} t^{\prime}$; then since $t \approx_{E}^{b^{\prime \prime}} u$ we have $u \stackrel{d_{j}, \hat{\alpha}}{\longrightarrow} u^{\prime} \approx_{E}^{b^{\prime \prime}} t^{\prime}$. By assumption $u^{\prime}$ can not be $u$ itself when $\alpha \equiv \tau$, so $u \stackrel{d_{j, \alpha}}{\longrightarrow} u^{\prime} \approx_{E}^{b^{\prime \prime}} t^{\prime}$ as required. By symmetry, $t \simeq_{E}^{b^{\prime \prime}} u$.

Now the required $B$ is $\cup_{b^{\prime} \in B^{\prime}} B_{b^{\prime}}$.

Finally, since we are considering E-INPUT rather than EA we need the following generalisation:

Proposition 4.3. Suppose $x \notin f v\left(b, c_{i}, d_{j}\right), i \in I, j \in J$. Then from

$$
\vdash_{1} b \triangleright \Sigma_{i \in I} c_{i} \rightarrow \tau . t_{i}=\Sigma_{j \in J} d_{j} \rightarrow \tau . u_{j}
$$

infer

$$
\vdash_{1} b \triangleright \Sigma_{i \in I} c_{i} \rightarrow c ? x . t_{i}=\Sigma_{j \in J} d_{j} \rightarrow c ? x . u_{j}
$$

Proof. For each $K \subseteq I$ let $c_{K}$ be the boolean expression $\bigwedge_{k \in K} c_{k} \wedge \wedge_{k^{\prime} \in I-K} \neg c_{k^{\prime}}$. Then $\bigvee c_{K}=$ true, $c_{K} \wedge c_{K^{\prime}}=$ false whenever $K \neq K^{\prime}$. Using parts 3,6 and 5 of Proposition 2.4 we can show that

$$
\vdash_{1} \Sigma_{i \in I} c_{i} \rightarrow \tau . t_{i}=t^{\tau}
$$

where $t^{\tau}$ denotes $\Sigma_{K} c_{K} \rightarrow t_{K}^{\tau}$ and $t_{K}^{\tau}$ denotes $\Sigma_{k \in K} c_{k} \rightarrow \tau . t_{k}$. Let $u^{\tau}=\Sigma_{L} d_{L} \rightarrow u_{L}^{\tau}$ be defined in a similar manner.

We know $\vdash_{1} b \triangleright t^{\tau}=u^{\tau}$ and therefore for each $K, L, \vdash_{1} b \wedge c_{K} \wedge d_{L} \triangleright t^{\tau}=u^{\tau}$. Again using parts 3 and 5 of Proposition 2.4 we can prove

$$
\vdash_{1} b \wedge c_{K} \wedge d_{L} \triangleright t^{\tau}=\Sigma_{k \in K} \tau . t_{k}
$$

and

$$
\vdash_{1} b \wedge c_{K} \wedge d_{L} \triangleright u^{\tau}=\Sigma_{l \in L} \tau . u_{l}
$$


Therefore

$$
\vdash_{1} b \wedge c_{K} \wedge d_{L} \triangleright \Sigma_{k \in K} \tau . t_{k}=\Sigma_{l \in L} \tau . u_{l}
$$

Now we can apply E-INPUT to obtain

$$
\vdash_{1} b \wedge c_{K} \wedge d_{L} \triangleright \Sigma_{k \in K} c ? x . t_{k}=\Sigma_{l \in L} c ? x . u_{l}
$$

By reversing the above argument we have

$$
\vdash_{1} b \wedge c_{K} \wedge d_{L} \triangleright t^{x}=u^{x}
$$

where $t^{x}$ and $u^{x}$ denote $\Sigma_{K} c_{K} \rightarrow \Sigma_{k \in K} c$ ?x. $t_{k}$ and $\Sigma_{L} d_{L} \rightarrow \Sigma_{l \in L} c$ ?x. $u_{l}$, respectively. Since $\bigvee_{K, L} c_{K} \wedge d_{L}=t r u e$, we can apply CUT to obtain $\vdash_{1} b \triangleright t^{x}=u^{x}$. Finally parts 3,6 and 5 of Proposition 2.4 can be used as above to transform $t^{x}$ and $u^{x}$ into the required form.

We now have the main technical ingredients necessary for the completeness proof. As usual this requires some notion of normal form, which we call full normal forms. For convenience we consider these before embarking on the completeness proof proper.

Definition 4.5. A normal form $t \equiv \Sigma_{i} b_{i} \rightarrow \alpha_{i} ._{i}$ is a full normal form if

1. $t \stackrel{b, \alpha}{\Longrightarrow} E t^{\prime}$, where $b v(\alpha) \cap f v(b)=\emptyset$, implies $t \stackrel{b, \alpha}{\longrightarrow} t^{\prime}$.

2. Each $t_{i}$ is in full normal form.

Lemma 4.9. For any normal form $t$ there is a full normal form $t^{\prime}$ such that $f v(t)=f v\left(t^{\prime}\right),|t|=\left|t^{\prime}\right|$ and $\vdash_{2} t=t^{\prime}$.

Proof. By structural induction on $t$. For then non-trivial case when $t \not \equiv n i l$, by induction we may assume each summand of $t$ is already in full normal form. Let

$$
t^{\prime}=t+\Sigma_{k}\left\{b_{k} \rightarrow \alpha_{k} \cdot t_{k} \mid t \stackrel{b_{k}, \alpha_{k}}{\Longrightarrow} t_{k}, b v\left(\alpha_{k}\right) \cap f v\left(b_{k}\right)=\emptyset \text {, but not } t \stackrel{b_{k}, \alpha_{k}}{\longrightarrow} t_{k}\right\}
$$

Then, modulo $\alpha$-equivalence, $t^{\prime}$ is in full normal form with size equal to $t$, and by the absorption lemma $\vdash_{2} t=t^{\prime}$.

By this lemma and Lemma 3.5, every term can be transformed into a full normal form of equal size.

Theorem 4.3. (Completeness of $\vdash_{2}$ ) $t \simeq_{E}^{b} u$ implies $\vdash_{2} b \triangleright t=u$.

Proof. We may assume $t, u$ are in full normal form and apply induction on the joint weak size of $t$ and $u$. The case that the size is 0 is trivial. So let $t \equiv \Sigma_{i \in I} c_{i} \rightarrow \alpha_{i} . t_{i}, u \equiv \Sigma_{j \in J} d_{j} \rightarrow \alpha_{j} . u_{j}$. We use the notations $I_{\gamma}, J_{\gamma}, t_{\gamma}, u_{\gamma}$ as defined in the proof of Theorem 3.2.

Consider the case $\gamma \equiv c$ ? . Let

$$
t_{c ?}^{\tau} \equiv \Sigma_{i \in I_{c} ?} c_{i} \rightarrow \tau . t_{i} \quad u_{c ?}^{\tau} \equiv \Sigma_{j \in J_{c} ?} d_{j} \rightarrow \tau . u_{j}
$$

Since $t \simeq_{E}^{b} u$, we have $t_{c ?} \simeq_{E}^{b} u_{c ?}$ and therefore $t_{c ?}^{\tau} \simeq_{E}^{b} u_{c}^{\tau}$.

To prove $\vdash_{2} b \triangleright t_{c}$ ? $=u_{c}$ ? it is sufficient to establish

$$
\vdash_{2} b \wedge c_{i} \triangleright u_{c}+c_{i} \rightarrow c ? x . t_{i}=u_{c} \text { ? }
$$

for each $i \in I_{c ?}$.

Now $t_{c} \stackrel{c_{i}, c ? x}{\longrightarrow} t_{i}$, so there is a $b \wedge c_{i}$-partition $B$ with the property that for each 
$b^{\prime} \in B$ there is $u_{c} \stackrel{d_{j}, c ? x}{\longrightarrow} u_{j} \stackrel{d, \hat{\tau}}{\Longrightarrow} u^{\prime}$ s.t. $b^{\prime} \models d_{j} \wedge d^{\prime}$ and $t_{i} \approx_{E}^{b^{\prime}} u^{\prime}$. By Proposition 4.2 there exists a $b^{\prime}$-partition $B^{\prime}$, for each $b^{\prime \prime} \in B^{\prime} t_{i} \simeq_{E}^{b^{\prime \prime}} u^{\prime}$ or $t_{i} \simeq_{E}^{b^{\prime \prime}}$ c.u or $\tau . t_{i} \simeq_{E}^{b^{\prime \prime}} u^{\prime}$. By induction, together with TAU and $\mathrm{T} 1$, in each case we can derive

$$
\vdash_{2} b^{\prime \prime} \triangleright \tau . u^{\prime}=\tau . t_{i}
$$

Applying CUT on $B^{\prime}$ we get $\vdash_{2} b^{\prime} \triangleright \tau . u^{\prime}=\tau . t_{i}$.

If $u_{j} \equiv u^{\prime}$, then $\vdash_{2} b^{\prime} \triangleright \tau . u_{j}=\tau . t_{i}$ and hence $\vdash_{2} b^{\prime} \triangleright \tau . u_{j}=\tau . u_{j}+\tau . t_{i}$.

Otherwise, by absorption $\vdash_{2} u_{j}=u_{j}+d^{\prime} \rightarrow \tau . u^{\prime}$. Therefore

$$
\begin{aligned}
\vdash_{2} b^{\prime} \triangleright \tau . u_{j} & =\tau .\left(u_{j}+d^{\prime} \rightarrow \tau . u^{\prime}\right) \\
& \stackrel{4.7}{=} \tau .\left(u_{j}+d^{\prime} \rightarrow \tau . u^{\prime}\right)+d^{\prime} \rightarrow \tau . u^{\prime} \\
& =\tau . u_{j}+d^{\prime} \rightarrow \tau . u^{\prime} \\
& =\tau . u_{j}+d^{\prime} \rightarrow \tau . t_{i}
\end{aligned}
$$

Since $b^{\prime} \models d^{\prime}$, by Proposition 2.4.3 it follows that

$$
\vdash_{2} b^{\prime} \triangleright \tau . u_{j}=\tau . u_{j}+\tau . t_{i}
$$

Similarly, because $b^{\prime} \models c_{i} \wedge d_{j}$, we have

$$
\vdash_{2} b^{\prime} \triangleright d_{j} \rightarrow \tau . u_{j}=d_{j} \rightarrow \tau . u_{j}+c_{i} \rightarrow \tau . t_{i}
$$

So

$$
\vdash_{2} b^{\prime} \triangleright u_{c ?}^{\tau}=u_{c ?}^{\tau}+c_{i} \rightarrow \tau . t_{i}
$$

This is true for each $b^{\prime}$ in the $b \wedge c_{i}$-partition $B$. So applying CUT we obtain $\vdash_{2} b \wedge c_{i} \triangleright u_{c ?}^{\tau}=u_{c ?}^{\tau}+c_{i} \rightarrow \tau . t_{i}$. By Proposition 4.3, the generalised E-INPUT Rule, applicable because we can assume $x \notin f v\left(b \wedge c_{i}\right)$, we get the required $\vdash_{2} b \wedge c_{i} \triangleright u_{c ?}+c_{i} \rightarrow \alpha_{i} \cdot t_{i}=u_{c}$.

\section{The Late Case}

In this section we briefly outline how the theory developed in the previous sections can be carried over to the late case with some systematic modifications. It turns out that only those parts concerning input actions need changing and for brevity we only treat weak equivalence.

The late operational semantics of this language is given in Fig. 6. Note that both $\stackrel{\tau}{\longrightarrow}$ and $\stackrel{c ! v}{\longrightarrow} l$ are relations over closed terms but $\stackrel{c ? x}{\longrightarrow}$ is a relation between closed terms and functions from values to closed terms. Intuitively $p \stackrel{c ? x}{\longrightarrow} \lambda x$. $u$ means that the process can accept inputs on channel $c$ and when it does so its future behaviour, which is parameterised on the value received, is characterised by the function $\lambda x . u$.

The concrete late double arrow relations are also defined in the same way as in the early case in Section 4 , with $\stackrel{a}{\Rightarrow}$ in place of $\stackrel{a}{\Longrightarrow}$, except that the last clause is only given for non-input actions; so input actions do not absorb $\tau$ moves after them:

- $p \stackrel{\varepsilon}{\Longrightarrow} 1 p$.

- $p \stackrel{a}{\longrightarrow} l q$ implies $p \stackrel{a}{\Longrightarrow} l q$.

- $p \stackrel{\tau}{\longrightarrow} \stackrel{a}{\longrightarrow} l q$ implies $p \stackrel{a}{\Longrightarrow} I q$. 


$$
\begin{array}{lll}
\tau . p \stackrel{\tau}{\longrightarrow} l p & \\
c ! e . p \stackrel{c ! v}{\longrightarrow} l p & \text { where } v=\llbracket e \rrbracket \\
c ? x . t \stackrel{c ? x}{\longrightarrow} l \lambda x . t & \\
p \stackrel{\alpha}{\longrightarrow} l r & \text { implies } & p+q \stackrel{\alpha}{\longrightarrow} l r \\
p \stackrel{\alpha}{\longrightarrow} l r, \llbracket b \rrbracket=\text { true } & \text { implies } & b \rightarrow p \stackrel{\alpha}{\longrightarrow} r
\end{array}
$$

Fig. 6. Late concrete operational semantics.

- $p \stackrel{\tau}{{ }_{\Longrightarrow}} \stackrel{\tau}{\longrightarrow} l q$ implies $p \stackrel{\tau}{\Longrightarrow} l q$.

- $p \stackrel{c ! v}{\Longrightarrow} \stackrel{\tau}{\longrightarrow} l q$ implies $p \stackrel{c ! v}{\Longrightarrow} q$.

These revised arrows can now be used to define another version of weak bisimulation equivalence.

Definition 5.1. A symmetric relation $R$ between closed terms is a late weak bisimulation if it satisfies: $(p, q) \in R$ implies

- If $p \stackrel{c ? x}{\longrightarrow} \lambda x . t$ then there exists $q \stackrel{c ? y}{\Longrightarrow} \lambda y . u$ and for all $v \in V a l \exists q^{\prime}$ s.t. $u[v / y] \stackrel{\varepsilon}{\Longrightarrow} l q^{\prime}$ and $\left(t[v / x], q^{\prime}\right) \in R$.

- For any other action $a$ not of the form $c ? x, p \stackrel{a}{\longrightarrow} l p^{\prime}$ then there exists $q \stackrel{\hat{a}}{\Longrightarrow} l q^{\prime}$ and $\left(p^{\prime}, q^{\prime}\right) \in R$.

Let $\approx_{l}$ be the largest late weak bisimulation.

The corresponding late observation congruence is then defined in terms of late weak bisimulation:

Definition 5.2. Two closed terms is $p, q$ are late observation congruent, written $p \simeq_{l} q$, if

- Whenever $p \stackrel{c ? x}{\longrightarrow} l \lambda x . t$ then there exists $q \stackrel{c ? y}{\Longrightarrow} \lambda y . u$ and for all $v \in V a l \exists q^{\prime}$ s.t. $u[v / y] \Longrightarrow_{l} q^{\prime}$ and $t[v / x] \approx_{l} q^{\prime}$.

- For any other action $a$, whenever $p \stackrel{a}{\longrightarrow} l p^{\prime}$ then there exists $q \stackrel{a}{\Longrightarrow} l q^{\prime}$ and $p^{\prime} \approx_{l}$ $q^{\prime}$.

- similarly for $q$.

This relation is generalised to open terms by letting $t \simeq_{l} u$ iff $t \rho \simeq_{l} u \rho$ for any $\rho$.

It can be shown that $\simeq_{l}$ is preserved by all operators in our language. In general it is finer that $\simeq_{e} ;$ a typical example of a distinction made by $\simeq_{l}$ but not by $\simeq_{e}$ is discussed in the Introduction.

To develop results for $\simeq_{l}$ similar to those in the previous section we need a symbolic version of late weak equivalence. This, called late weak symbolic equivalence, is defined using a corresponding notion of late weak symbolic action:

- $t \stackrel{\text { true,s }}{\Longrightarrow} t$.

- $t \stackrel{b, \alpha}{\longrightarrow} u$ implies $t \stackrel{b, \alpha}{\Rightarrow} L u$.

- $t \stackrel{b, \tau}{\longrightarrow} \stackrel{b^{\prime}, \alpha}{\Longrightarrow} L$ implies $t \stackrel{b \wedge b^{\prime}, \alpha}{\Longrightarrow} u$. 
- if $\alpha$ is not of the form $c$ ? $x$ then $t \stackrel{b, \alpha}{\Longrightarrow} L \stackrel{b^{\prime}, \tau}{\longrightarrow} u$ implies $t \stackrel{b \wedge b^{\prime}, \alpha}{\Longrightarrow} u$.

The difference between the last clause and the corresponding one in the definition of early symbolic double arrows in Section 4 is important. Now we do not have Lemma 4.2. Instead the following holds

Lemma 5.1. If $t \stackrel{b, c^{9} x}{=} t_{E}^{\prime}$ then $f v(b) \subseteq f v(t)$ and $x \notin f v(t)$.

Definition 5.3. A family of symmetric relations $\mathbf{S}=\left\{S^{b} \mid b \in B \operatorname{Exp}\right\}$ is a late weak symbolic bisimulation if:

$(t, u) \in S^{b}$ implies whenever $t \stackrel{b_{1}, \alpha}{\longrightarrow} t^{\prime}$ with $b v(\alpha) \cap f v(b, t, u)=\emptyset$, then there is a $b \wedge b_{1}$-partition $B$ such that $f v(B) \subseteq f v(b, t, u)$ and for each $b^{\prime} \in B$ there exists a $u \stackrel{b_{2}, \hat{\alpha}^{\prime}}{\Rightarrow} L u^{\prime}$ such that $b^{\prime} \models b_{2}$ and

1. if $\alpha \equiv c ! e$ then $\alpha^{\prime} \equiv c ! e^{\prime}, b^{\prime}=e=e^{\prime}$ and $\left(t^{\prime}, u^{\prime}\right) \in S^{b^{\prime}}$.

2. if $\alpha \equiv \tau$ then $\alpha^{\prime} \equiv \tau$ and $\left(t^{\prime}, u^{\prime}\right) \in S^{b^{\prime}}$.

3. if $\alpha \equiv c$ ? $x$ then $\alpha^{\prime} \equiv c$ ? $x$ and there is a $b^{\prime}$-partition $B^{\prime}$ s.t for each $b^{\prime \prime} \in B^{\prime}$ there is $u^{\prime} \stackrel{b_{2}^{\prime}, \hat{\tau}}{\Longrightarrow} u_{L}^{\prime \prime}$ s.t $b^{\prime \prime} \models b_{2}^{\prime}$ and $\left(t^{\prime}, u^{\prime \prime}\right) \in S^{b^{\prime \prime}}$.

Let $\approx_{\mathbf{L}}=\left\{\approx_{L}^{b}\right\}$ be the largest late weak symbolic bisimulation.

It is important to note that we now require $f v(B) \subseteq f v(b, t, u)$; hence when $\alpha \equiv c ? x$ it is guaranteed that $x \notin f v(B)$. So we can not partition over the value space for an input variable. This makes all the differences between early and late bisimulations!

Late weak symbolic observation congruence is defined in terms of weak symbolic bisimulation:

Definition 5.4. Two terms $t, u$ are late weak symbolic observation congruent over $b$, written $t \simeq_{L}^{b} u$, if whenever $t \stackrel{b_{1}, \alpha}{\longrightarrow} t^{\prime}$ with $b v(\alpha) \cap f v(b, t, u)=\emptyset$, then there is a $b \wedge b_{1}$-partition $B$ such that $f v(B) \subseteq f v(b, t, u)$ and for each $b^{\prime} \in B$ there exists a $u \stackrel{b_{2}, \alpha^{\prime}}{\Longrightarrow} L u^{\prime}$ such that $b^{\prime}=b_{2}$ and

1. if $\alpha \equiv c$ !e then $\alpha^{\prime} \equiv c ! e^{\prime}, b^{\prime}=e=e^{\prime}$ and $t^{\prime} \approx_{L}^{b^{\prime}} u^{\prime}$.

2. if $\alpha \equiv \tau$ then $\alpha^{\prime} \equiv \tau$ and $t^{\prime} \approx_{L}^{b^{\prime}} u^{\prime}$.

3. if $\alpha \equiv c ? x$ then $\alpha^{\prime} \equiv c ? x$ and there is a $b^{\prime}$-partition $B^{\prime}$ s.t for each $b^{\prime \prime} \in B^{\prime}$ there is $u^{\prime} \stackrel{b_{2}^{\prime}}{\Longrightarrow} u^{\prime \prime}$ s.t $b^{\prime \prime} \models b_{2}^{\prime}$ and $t^{\prime} \approx_{L}^{b^{\prime \prime}} u^{\prime \prime}$.

and symmetrically for $u$.

We have the late counterpart of Theorem 4.2:

Theorem 5.1. (Soundness and completeness of $\approx_{L}$ and $\simeq_{L}$ )

1. $t \approx_{L}^{b} u$ if and only if $t \rho \approx_{l} u \rho$ for every $\rho \models b$.

2. $t \simeq_{L}^{b} u$ if and only if $t \rho \simeq_{l} u \rho$ for every $\rho \models b$.

Proof. Similar to that of Theorems 4.2. The only essential difference is in the relationship between the abstract moves, $\stackrel{b, \alpha}{\longrightarrow}$ and the concrete moves, $\stackrel{\alpha}{\longrightarrow}$. This is the same as for the early moves, given in Lemma $3.2-3.4$ and $4.3-4.6$, except for input actions now we have: 
$t \rho \stackrel{c ? x}{\longrightarrow} \lambda x . u$ if and only if there exists some $b, t^{\prime}$ such that $\rho \models b$ and $t \stackrel{b, c ? z}{\longrightarrow} t^{\prime}$ for some $z$ where $u \equiv t^{\prime}[x / z]$.

The strong version of the theorem is now essentially the same as Theorem 4.5 of [HeL92] and the weak version is a very straightforward extension.

The inference system for late symbolic observation congruence can be obtained by deleting EA/E-INPUT from that for early congruence. We write $\vdash_{2 L} b \triangleright t=u$ to denote $b \triangleright t=u$ can be derived from the new inference system. We have the soundness theorem:

Theorem 5.2. (Soundness of $\vdash_{2 L}$ ) $\vdash_{2 L} b \triangleright t=u$ implies $t \rho \simeq_{l} u \rho$ for every $\rho$ such that $\rho \models b$.

For the completeness theorem, we use essentially the same form of full normal form as in the early case (keep in mind that now double input arrows only absorb those $\tau$ moves before it):

Definition 5.5. A normal form $t \equiv \Sigma_{i} b_{i} \rightarrow \alpha_{i} . t_{i}$ is a late full normal form if

1. $t \stackrel{b, \alpha}{\Longrightarrow} t^{\prime}$ implies $t \stackrel{b, \alpha}{\longrightarrow} t^{\prime}$.

2. Each $t_{i}$ is in late full normal form.

The Absorption Lemma, Lemma 4.8, still holds (but note that now $\alpha$ can not be an input action in the second case in the proof of the lemma) and therefore every term can be transformed to late normal form. Also the appropriate version of Proposition 4.2 holds.

Theorem 5.3. (Completeness of $\vdash_{2 L}$ ) $t \simeq_{L}^{b} u$ implies $\vdash_{2 L} b \triangleright t=u$.

Proof. We assume $t, u$ are in late full normal form and the proof proceeds by induction on the joint weak size of $t$ and $u$. For the non-trivial case when the size is not 0 let $t \equiv \Sigma_{i \in I} c_{i} \rightarrow \alpha_{i} . t_{i}, u \equiv \Sigma_{j \in J} d_{j} \rightarrow \beta_{j} . u_{j}$. We need to show

$$
\vdash_{2 L} b \wedge c_{i} \triangleright u+c_{i} \rightarrow \alpha_{i} \cdot t_{i}=u
$$

for each $i \in I$. We only consider the case when $\alpha_{i} \equiv c$ ? $x$ here (the other two cases are the same as in the early case). By $\alpha$-CONV we may assume $x \notin f v(b, t, u)$ and every input prefix in $u$ uses $x$ as input variable.

Now $t \stackrel{c_{i}, c ? x}{\longrightarrow} t_{i}^{\prime}$. So there exists a $b \wedge c_{i}$-partition $B$ with $f v(B) \subseteq f v\left(b \wedge c_{i}\right)$ s.t for all $b^{\prime} \in B, b^{\prime} \models c_{i}$ and there is $u \stackrel{d_{j}, c \text { ?x }}{\longrightarrow} u_{j}$ s.t. $b^{\prime} \models d_{j}$ and there exists a $b^{\prime}$-partition $B^{\prime}$ s.t for all $b^{\prime \prime} \in B^{\prime}$ there is $u_{j} \stackrel{d^{\prime}, \hat{\tau}}{\Longrightarrow} u^{\prime}$ s.t. $b^{\prime \prime} \models d^{\prime}$ and $t_{i} \approx_{L} u^{\prime}$.

By Proposition 4.2 and induction, together with TAU and T1, we can derive, for each $b^{\prime \prime} \in B$,

$$
\vdash_{2 L} b^{\prime \prime} \triangleright \tau . u^{\prime}=\tau . t_{i}
$$

By an argument similar to that used in Theorem 4.3, using CUT on $B^{\prime}$, we obtain

$$
\vdash_{2 L} b^{\prime} \triangleright \tau . u_{j}=\tau . u_{j}+\tau . t_{i}
$$

Now, since $x \notin f v\left(b^{\prime}\right)$, we can apply INPUT to get

$$
\begin{aligned}
\vdash_{2 L} b^{\prime} \triangleright c ? x . \tau . u_{j} & =c ? x .\left(\tau . u_{j}+\tau . t_{i}\right) \\
& \stackrel{T 3}{=} c ? x \cdot\left(\tau . u_{j}+\tau . t_{i}\right)+c ? x . t_{i} \\
& =c ? x . \tau . u_{j}+c ? x . t_{i}
\end{aligned}
$$




$$
\begin{array}{lll}
t \stackrel{b, \alpha}{\longrightarrow} t^{\prime} & \text { implies } & t\left|u \stackrel{b, \alpha}{\longrightarrow} t^{\prime}\right| u \\
& & \alpha \in\{\tau, c ! e \mid c \in \text { Chan, } e \in \operatorname{Exp}\} \\
t \stackrel{b, c ? x}{\longrightarrow} t^{\prime} & \text { implies } & t\left|u \stackrel{b, c ? x}{\longrightarrow} t^{\prime}\right| u \\
& x \notin f v(u) \\
t \stackrel{b, c ? x}{\longrightarrow} t^{\prime}, u \stackrel{b^{\prime}, c ! e}{\longrightarrow} u^{\prime} & \text { implies } & t\left|u \stackrel{b \wedge b^{\prime}, \tau}{\longrightarrow} t^{\prime}[e / x]\right| u^{\prime} \\
t \stackrel{b, \alpha}{\longrightarrow} t^{\prime} & \text { implies } & t \backslash c \stackrel{b, \alpha}{\longrightarrow} t^{\prime} \backslash c \\
& & \text { if } \operatorname{chan}(\alpha) \neq c
\end{array}
$$

Fig. 7. Symbolic operational semantics - continued.

$$
\begin{aligned}
& \text { nil } \backslash c=n i l \\
& (X+Y) \backslash c=X \backslash c+Y \backslash c \\
& (\alpha . X) \backslash c= \begin{cases}\alpha .(X \backslash c) & \text { if } \operatorname{chan}(\alpha) \neq c \\
\text { nil } & \text { if } \operatorname{chan}(\alpha)=c\end{cases}
\end{aligned}
$$

Let $X, Y$ denote $\Sigma_{i} \alpha_{i} . X_{i}, \Sigma_{j} \beta_{j} . Y_{j}$, with $f v(X) \cap b v(Y)=f v(Y) \cap b v(X)=\emptyset$ where $f v(X)$ and $b v(X)$ are free data variables and bound data variables in the term $X$, respectively. Then

$$
\begin{aligned}
& X \mid Y=\operatorname{sync} \text { move }(X, Y)+\operatorname{async\_ move}(X, Y) \\
& \text { where } \\
& \operatorname{sync\_ move}(X, Y)=\sum\left\{\tau .\left(X_{i}\{e / x\} \mid Y_{j}\right) \mid \alpha_{i} \equiv c ? x, \beta_{j} \equiv c ! e\right\}+ \\
& \Sigma\left\{\tau .\left(X_{i} \mid Y_{j}\{e / x\}\right) \mid \alpha_{i} \equiv c ! e, \beta_{j} \equiv c ? x\right\} \\
& \text { async.move }(X, Y)=\Sigma_{i} \alpha_{i} \cdot\left(X_{i} \mid Y\right)+\Sigma_{j} \beta_{j} .\left(X \mid Y_{j}\right)
\end{aligned}
$$

Fig. 8. New equations and expansion law.

By $\mathrm{T} 1, \vdash_{2 L} b^{\prime} \triangleright c ? x . u_{j}=c ? x . u_{j}+c ? x . t_{i}$. Since $b^{\prime} \models c_{i} \wedge d_{j}$, we can derive $\vdash_{2 L} b^{\prime} \triangleright d_{j} \rightarrow c ? x . u_{j}=d_{j} \rightarrow c ? x . u_{j}+c_{i} \rightarrow c ? x . t_{i}$. Hence $\vdash_{2 L} b^{\prime} \triangleright u=u+c_{i} \rightarrow c ? x . t_{i}$. Finally, an application of CUT on $B$ gives the required $\vdash_{2 L} b \wedge c_{i} \triangleright u=u+c_{i} \rightarrow$ $c ? x . t_{i}$.

\section{Extensions}

So far we have concentrated on the core language of Section 2. As mentioned in the Introduction all our rsults can be easily extended to the language obtained by adding the $\mid$ (parallel) and $\backslash$ (restriction) operators. The concrete operational semantics for these operators are standard and we only give their symbolic operational semantics, in Fig. 7, where symmetric rules have been omitted. The equations characterizing the restriction operator and the expansion law for the parallel operator are shown in Fig. 8. These laws are fairly standard and are only included here just for the sake of completeness.

It is routine to check that all $\sim_{e}, \simeq_{e}, \sim_{l}$ and $\simeq_{l}$ are preserved by the new operators, and that the new equations are sound for these congruence relations. Moveover these new equations are sufficient to reduce every term in the extended language to one in the core language. 
Now if we add these new equations to $\mathscr{A}_{1}$, then the three normal form lemmas 3.5, 4.9 and 5.5 carry over to the extended language. From these follow the completeness results (Theorem 3.2, 4.3 and 5.3) for the extended language.

\section{Acknowledgements}

The authors would like to thank Luca Aceto for carefully reading a draft of this paper and suggesting many improvements, and also two anonymous referees for their the valuable comments. This work has been supported by the SERC grant GR/H16537 and the ESPRIT BRA project CONCUR II.

\section{References}

[Bur91] Burns, G. A language for value-passing ces. Technical Report ECS-LFCS-91-175, University of Edinburgh, August 1991.

[CPS89] Cleaveland, R., Parrow, J., Steffen, B. The concurrency workbench: A semantics based verification tool for finite state systems. ACM Transactions on Programming Systems, 15:36-72, 1989.

[GrP90] Groote, J.F., Ponse, A. The syntax and semantics of $\mu$ CRL. Technical Report CS-R9076, CWI, Amsterdam, 1990.

[GrP91] Groote, J.F., Ponse, A. Proof theory for $\mu$ CRL. Technical Report CS-R9138, CWI, Amsterdam, 1991.

[Hen88] Hennessy, M. An Algebraic Theory of Processes. MIT Press, 1988.

[Hen91] Hennessy, M. A proof system for communicating processes with value-passing. Formal Aspects of Computer Science, 3:346-366, 1991.

[HeI93] Hennessy, M., Ingolfsdottir, A. A theory of communicating processes with value-passing. Information and Computation, 107(2):202-236, 1993.

[HeL92] Hennessy, M. Lin, H. Symbolic bisimulations. Theoretical Computer Science, 138:353389,1995 .

[HeL95] Hennessy, M. Liu, X. A modal logic for message passing processes. Acta Informatica, 32:375-393, 1995.

[HoR86] Hoare, C.A.R., Roscoe, A.W. The laws of occam. Technical Report PRG Monograph 53, Oxford University Computing Laboratory, 1986.

[Lin91] Lin, H. PAM: A process algebra manipulator. In K. Larsen and A. Skou, editors, Computer Aided Verification, volume 575 of Lecture Notes in Computer Science, pages 136-146. Springer-Verlag, 1991. Also available as Sussex Computer Science Technical Report 9/91.

[Lin93] Lin, H. A verification tool for value-passing processes. In Proceedings of $13^{\text {th }}$ International Symposium on Protocol Specification, Testing Verification, IFIP Transactions. North-Holland, 1993.

[Mil89] Milner, R. Communication and Concurrency. Prentice-Hall, 1989.

[MPW92] Milner, R., Parrow, J., Walker, D. A calculus of mobile proceses, part i. Information and Computation, 100(1):1-40, 1992.

[PaS93] Parrow, J., Sangiorgi, D. Algebraic theories for value-passing calculi. Technical report, University of Edinburgh, 1993. Also Technical Report from SICS, Sweden. To appear in Information and Computation.

[Wal89] Walker, D. Automated analysis of mutual exclusion algorithms using CCS. Formal Aspects of Computing, 1:273-292, 1989.

Received June 1994

Accepted in revised form August 1995 by J. Parrow 